\title{
BMJ Open Standards for Reporting Implementation Studies (StaRI): explanation and elaboration document
}

\author{
Hilary Pinnock, ${ }^{1}$ Melanie Barwick, ${ }^{2,3}$ Christopher R Carpenter, ${ }^{4}$ Sandra Eldridge,${ }^{5}$ \\ Gonzalo Grandes, ${ }^{6}$ Chris J Griffiths, ${ }^{7}$ Jo Rycroft-Malone, ${ }^{8}$ Paul Meissner, ${ }^{9}$ \\ Elizabeth Murray, ${ }^{10}$ Anita Patel, ${ }^{7}$ Aziz Sheikh, ${ }^{1}$ Stephanie J C Taylor, ${ }^{7}$ for the StaRI \\ Group
}

To cite: Pinnock $\mathrm{H}$, Barwick M, Carpenter CR, et al. Standards for Reporting Implementation Studies (StaRI): explanation and elaboration document. BMJ Open 2017;7:e013318. doi:10.1136/bmjopen-2016013318

- Prepublication history for this paper is available online. To view these files please visit the journal online (http://dx.doi.org/10.1136/ bmjopen-2016-013318).

Received 5 July 2016 Revised 9 January 2017 Accepted 11 January 2017

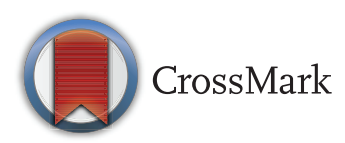

For numbered affiliations see end of article.

Correspondence to Professor Hilary Pinnock; hilary.pinnock@ed.ac.uk

\section{ABSTRACT}

Objectives: Implementation studies are often poorly reported and indexed, reducing their potential to inform the provision of healthcare services. The Standards for Reporting Implementation Studies (StaRI) initiative aims to develop guidelines for transparent and accurate reporting of implementation studies.

Methods: An international working group developed the StaRI guideline informed by a systematic literature review and e-Delphi prioritisation exercise. Following a face-to-face meeting, the checklist was developed iteratively by email discussion and critical review by international experts.

Results: The 27 items of the checklist are applicable to the broad range of study designs employed in implementation science. A key concept is the dual strands, represented as 2 columns in the checklist, describing, on the one hand, the implementation strategy and, on the other, the clinical, healthcare or public health intervention being implemented. This explanation and elaboration document details each of the items, explains the rationale and provides examples of good reporting practice.

Conclusions: Previously published reporting statements have been instrumental in improving reporting standards; adoption by journals and authors may achieve a similar improvement in the reporting of implementation strategies that will facilitate translation of effective interventions into routine practice.

Implementation science bridges the gap between developing and evaluating effective interventions and implementation in routine practice to improve patient and population health. ${ }^{1}$ Implementation studies are however often poorly reported and indexed, ${ }^{23}$ reducing their potential to inform the provision of healthcare services and improve health outcomes. ${ }^{4}$ The Standards for Reporting Implementation Studies (StaRI) initiative aimed to develop standards for transparent and accurate reporting of implementation studies. The StaRI statement describing the scope and

\section{Strengths and limitations of this study}

- We followed recommended methodology for developing health research reporting guidelines, including a literature review, an e-Delphi exercise, an international face-to-face consensus meeting and inviting expert feedback on draft versions of the Standards for Reporting Implementation Studies (StaRI) checklist.

- Implementation science is a broad field, and although the e-Delphi, working group and expert feedback enabled input from experts from a range of implementation science-related disciplines, we may have missed some perspectives.

- Distance and financial constraints limited the geographical spread of representatives at the face-to-face consensus working group, but we invited feedback on the penultimate draft from experts from across the world.

- Although our initial feedback suggests general agreement with the underlying concepts, the StaRI guidelines will need to be refined in the light of authors' and editors' practical experience of using the checklist.

conceptual underpinning is published in the $B M j 5^{5}$ this elaboration document provides detailed explanation of the individual items.

\section{METHODS}

Following established guidelines, ${ }^{6} 7$ we convened a consensus working group in London at which 15 international multidisciplinary delegates considered candidate items identified by a previous systematic literature review and an international e-Delphi prioritisation exercise, ${ }^{8}$ in the context of other published reporting standards and the panel's expertise in implementation science. The resultant checklist was subsequently developed iteratively by email discussion, and feedback on the penultimate draft guideline sought from colleagues working in implementation science. 


\section{Scope of the StaRI reporting standards}

Implementation research is the scientific study of methods to promote the systematic uptake of evidence-based interventions into practice and policy and hence improve health. $^{9-11}$ The discipline encompasses a broad range of methodologies applicable to improving the dissemination and implementation of clinical, healthcare, global health and public health interventions. ${ }^{12-14}$ The StaRI checklist focuses primarily on standards for reporting studies that evaluate implementation strategies developed to enhance the adoption, implementation and sustainability of interventions, ${ }^{15}$ but some items may be applicable to other study designs used in implementation science.

\section{The StaRI reporting guidelines}

Unlike most reporting guidelines that apply to a specific research methodology, StaRI is applicable to the broad range of study designs employed in implementation science. Authors are referred to other reporting standards for advice on reporting specific methodological features. In an evolving field, in which there is a range of study designs, terminology is neither static nor used consistently. ${ }^{16}$ For clarity, we have adopted specific terms in this paper; table 1 defines these terms and lists some of the alternative or related terminology.
Underpinning the StaRI reporting standards are the dual strands of describing, on the one hand, the implementation strategy and, on the other, the clinical, healthcare or public health intervention being implemented. ${ }^{17}$ These strands are represented as two columns in the checklist (see table 2). The primary focus of implementation science is the implementation strategy ${ }^{15}$ (column 1) and the expectation is that this will always be fully completed. The impact of the intervention on the target population should always be considered (column 2) and either health outcomes reported or robust evidence cited to support a known beneficial effect of the intervention on the health of individuals or populations. While all items are worthy of consideration, not all items will be applicable to or feasible in every study; a fully completed StaRI checklist may thus include a number of 'not applicable' items. For example, studies simultaneously testing a clinical intervention and an implementation strategy (Hybrid type 2 designs) would need to fully address both strands, whereas studies testing a clinical intervention while gathering information on its potential for implementation (Hybrid type 1) or testing an implementation strategy while observing the clinical outcomes (Hybrid Type 3) would focus primarily on items in the clinical intervention or implementation strategy columns, respectively. ${ }^{14}$

\section{ELABORATION ON INDIVIDUAL CHECKLIST ITEMIS}

\section{Item 1. Title}

Identification as an implementation study, and description of the methodology in the title and/or keywords

\section{Examples - Titles}

Accessibility, clinical effectiveness, and practice costs of providing a telephone option for routine asthma reviews: phase IV controlled implementation study. ${ }^{18}$

Adaptive Implementation of Effective Programs Trial (ADEPT): cluster randomized SMART trial comparing a standard versus enhanced implementation strategy to improve outcomes of a mood disorders program. ${ }^{19}$

\section{Explanation}

In addition to specifying the study design used (eg, cluster RCT, controlled before-and-after study, mixed-methods, economic evaluation, etc), it is important to identify the work explicitly as an implementation study, so that indexers, readers and systematic reviewers can easily identify relevant studies. The study design and 'implementation study' should both be included as key words and in the abstract.

\section{Item 2. Abstract}

Identification as an implementation study, including a description of the implementation strategy to be tested, the evidence-based intervention being implemented, and defining the key implementation and health outcomes.

\section{Examples - Abstracts}

Background: Attendance for routine asthma reviews is poor. A recent randomised controlled trial found that telephone consultations can cost-effectively and safely enhance asthma review rates...

Design of study: Phase IV controlled before-and-after implementation study.

Setting: A large UK general practice.

Method: Using existing administrative groups, all patients with active asthma $(n=1809)$ received one of three asthma review services: structured recall with a telephone-option for reviews versus structured recall with face-to-face-only reviews, or usual-care (to assess secular trends). Main outcome measures were: proportion of patients with active asthma reviewed within the previous 15 months... mode of review, enablement, morbidity, and costs to the practice. ${ }^{18}$

Background: Good quality evidence has been summarised into guideline recommendations to show that peri-operative fasting times could be considerably shorter than patients currently experience. The objective of this trial was to evaluate the effectiveness of three strategies for the implementation of recommendations about peri-operative fasting. 
Methods: A pragmatic cluster randomised trial underpinned by the PARIHS framework was conducted during 2006 to 2009 with a national sample of UK hospitals using time series with mixed methods process evaluation and cost analysis. Hospitals were randomised to one of three interventions: standard dissemination (SD) of a guideline package, SD plus a web-based resource championed by an opinion leader, and SD plus plan-do-study-act (PDSA). The primary outcome was duration of fluid fast prior to induction of anaesthesia. Secondary outcomes included duration of food fast, patients' experiences, and stakeholders' experiences of implementation, including influences. ANOVA was used to test differences over time and interventions.

Results: Nineteen acute NHS hospitals participated. Across timepoints, 3,505 duration of fasting observations were recorded. No significant effect of the interventions was observed for either fluid or food fasting times. The effect size was 0.33 for the web-based intervention compared to SD alone for the change in fluid fasting and was 0.12 for PDSA compared to SD alone. The process evaluation showed different types of impact, including changes to practices, policies, and attitudes. A rich picture of the implementation challenges emerged, including interprofessional tensions and a lack of clarity for decision-making authority and responsibility. ${ }^{20}$

\section{Explanation}

For clarity of indexing and identification, the abstract should state clearly the study design and identify the work as an implementation study. In line with the concept of dual strands that underpins the StaRI checklist, the implementation strategy and the evidence-based intervention being implemented should be described. Other important information that should be included are the context, implementation outcomes, resource use and, if appropriate, health intervention outcomes.

\section{Item 3. Introduction (Identify the problem)}

Description of the problem, challenge or deficiency in healthcare or public health that the intervention being implemented aims to address.

\section{Examples - Identify the problem}

In the U.S., a substantial percentage of morbidity and mortality (about 37\%) is related to four unhealthy behaviors: tobacco use, unhealthy diet, physical inactivity, and risky alcohol use... Primary care clinicians have many opportunities to assist their patients in modifying unhealthy behaviors; however, they are hampered by inadequate time, training, and delivery systems. ${ }^{21}$

Despite significant morbidity, attendance for routine asthma reviews is poor... Telephone consultations offer alternative access to routine asthma reviews, although a recent UK ruling decreed that the evidence base for this approach in asthma care was 'insufficient'. ${ }^{18}$

\section{Explanation}

Identifying and characterising the problem or deficiency that the intervention was designed to address may require data on, for example, the epidemiology of the condition, its impact on individuals or healthcare resources and evidence of a 'research to practice' gap (eg, actual performance rates). Characterising the challenge for implementation requires a description of the context in which the intervention will be implemented. This should include a summary of the key factors that might affect successful implementation in terms of the wider context (eg, governmental policies, major philosophical paradigms influencing decision makers, availability of resources) as well as barriers and enablers within the organisation and at individual professional level. $^{9}$

\section{Item 4. Introduction (Rationale: implementation strategy and intervention)}

The scientific background and rationale for the implementation strategy (including any underpinning theory/framework/model, how it is expected to achieve its effects and any pilot work).

\section{Examples}

\section{Rationale for the implementation strategy}

Facilitated rapid-cycle quality-improvement techniques (plan-do-study-act cycles [PDSA]) and learning collaboratives are effective in primary care settings, and the two strategies ought to be complementary. ${ }^{21}$

The Health Decision Model, which combines decision analysis, behavioral decision theory, and health beliefs, is useful to identify patient characteristics related to treatment adherence and subsequent blood pressure control... Successful implementation generally requires a comprehensive approach, in which barriers and facilitators to change in a specific setting are targeted. $^{22}$
The scientific background and rationale for the intervention being implemented (including evidence about its effectiveness and how it is expected to achieve its effects).

\section{Rationale for the intervention}

... brief interventions delivered in primary care office settings have affected smoking cessation and alcohol consumption. Although less evidence supports brief interventions for improving diet or increasing exercise, there are reasons for optimism. ${ }^{21}$

If not properly controlled, elevated blood pressure (BP) can lead to serious patient morbidity and mortality... Inconsistent patient adherence to the prescribed treatment regimen is known to contribute to poor rates of $\mathrm{BP}$ control and improving medication adherence has been shown to be effective in improving $\mathrm{BP} .^{22}$ 


\section{Explanation}

Authors of implementation studies need to explain the rationale for the choice of implementation strategy and for the validity of the intervention being implemented:

- The implementation strategy and underpinning 'logic pathway' will be described in detail in the Methods section (see item 12 for description and table 1 for definition and alternative terminology), but it is likely to be appropriate in the introduction to identify the approach used with supporting evidence for the choice of implementation theory/model/framework adopted (see first example: plan-do-study-act cycles ${ }^{21}$ ) and/or any pilot work or examples from other clinical areas or contexts. It will be important to show how the implementation strategy has been adapted to fit the context.

- The expectation is that there will be (ideally robust) evidence for the intervention (see second example: improving adherence improves BP control which reduces morbidity ${ }^{22}$ ). It is important that the strength of evidence is made explicit at the outset, ${ }^{23}$ especially as sometimes there is pressure to implement an intervention before the evidence base is fully developed (eg, for political imperatives). This will allow a judgement as to whether it is reasonable to assume that effective implementation will have health benefits or whether it is necessary to also assess health outcomes. Effective implementation of some interventions may have such incontrovertible evidence of benefit (eg, reducing smoking prevalence) that a health outcome may be unnecessary. Even when evidence is strong, the possibility that the impact of an intervention may be attenuated when it is implemented in routine practice needs to be considered.

It is recommended that reporting the methods, outcomes and conclusions related to the implementation strategy precedes the corresponding reporting of the health outcomes of the intervention (because the key question in an implementation study is about the impact of the implementation strategy). However, authors may wish to reverse this in the introduction and establish that the intervention is effective before explaining the approach they took to implementing it. The use of hybrid study designs, which combine features of clinical effectiveness and implementation studies, may affect the relative emphasis that is placed on the implementation and health intervention aspects of trials. ${ }^{14}$

\section{Item 5. Aims and objectives}

The aims of the study, differentiating between implementation objectives and any intervention objectives.

\section{Example - Aim}

The aim of our study is to evaluate the process and effectiveness of supported self-management (SMS) implemented as an integral part of the care for patients with type 2 diabetes mellitus provided by practice nurses. We will simultaneously address the following research questions:

1. What is the uptake of the SMS programme by the practice nurses, and what barriers hamper the implementation of SMS in routine primary care?

2. What is the effectiveness of SMS in terms of daily functioning, emotional health status, social participation, self-management behaviour, and health care use by patients with type 2 diabetes? ${ }^{24}$

\section{Explanation}

The aims and objectives should distinguish between the aim(s) of the implementation strategy and the aim(s) of the evidence-based intervention that is being implemented, possibly using two specific research questions as in the example. ${ }^{24}$ The aim of the intervention may be implicit if there is already strong evidence to support the health benefits of the intervention (eg, reducing smoking prevalence).

\section{Item 6. Methods: study design}

The design and key features of the evaluation (cross referencing to any appropriate methodology reporting standards) and any changes to study protocol, with reasons

Examples - Study design

The trial was designed as an implementation study with a before and after analysis. ${ }^{25}$

Implementation of Perioperative Safety Guidelines is a multicenter study in nine hospitals using an one-way (unidirectional) cross-over cluster trial design... It is impossible to deliver such a strategy simultaneously to all hospitals because of logistical, practical, and financial reasons. For that reason, a stepped wedge cluster randomized trial design is chosen. ${ }^{26}$

\section{Explanation}

The study design should be identified and the rationale explained. Any important changes to the study protocol should be described (or the absence of changes confirmed).

In contrast to most reporting standards, StaRI is applicable to a broad range of study designs, for example, cluster RCTs, controlled clinical trials, interrupted time series, cohort, case study, before and after studies, as well as mixed methods quantitative/qualitative assessments. ${ }^{2}$ A hierarchy of study design has been suggested in the context of studies implementing asthma self-management. ${ }^{4}$ Reporting standards exist for many of these designs such as cluster RCTs, ${ }^{27}$ pragmatic RCTs, ${ }^{28}$ observational studies, ${ }^{29}$ including use of routine data, ${ }^{30}$ non-randomised public health interventions, ${ }^{31}$ qualitative studies, ${ }^{32}$ as well as templates for describing interventions ${ }^{33}$ and local quality improvement initiatives. ${ }^{34}$ The StaRI checklist does not, therefore, include items related to specific design features (eg, randomisation, blinding, intracluster correlation, matching criteria for cohorts, data saturation). Authors are referred to appropriate methodological guidance on reporting these aspects of their study (available from http://www.equator-network.org). 
Table 1 Terminology, definitions and resources

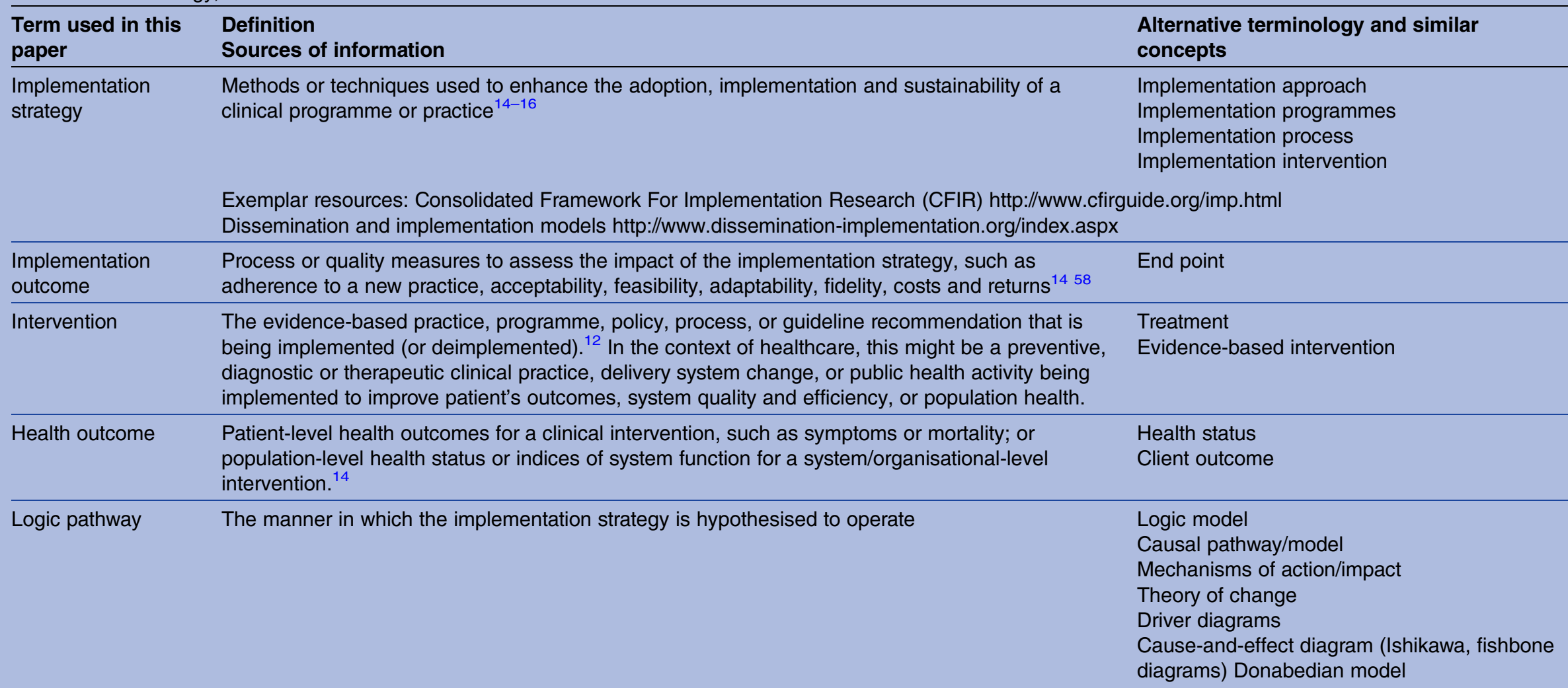

Exemplar resource: Logic models: https:/www.wkkf.org/resource-directory/resource/2006/02/wk-kellogg-foundation-logic-model-development-guide

\begin{tabular}{|c|c|c|}
\hline \multirow[t]{2}{*}{ Process evaluation } & $\begin{array}{l}\text { A study that aims to understand the functioning of an intervention, by examining its } \\
\text { implementation, mechanisms of impact and contextual factors. Process evaluation is } \\
\text { complementary to, but not a substitute for, high quality outcomes evaluation } \\
\text { Process evaluation aims to describe the strategy for change as planned, the strategy as delivered, } \\
\text { the actual exposure of the target population to the activities that are part of the strategy, and the } \\
\text { experiences of the people exposed } \\
\text { (Formative evaluation) is a rigorous assessment process designed to identify potential and actual } \\
\text { influences on the progress and effectiveness of implementation efforts }{ }^{62}\end{array}$ & Formative evaluation \\
\hline & $\begin{array}{l}\text { Exemplar resource: process evaluation of complex interventions. Available from https://www.ioe.ac.u } \\
\text { final(2).pdf }\end{array}$ & IK/MRC_PHSRN_Process_evaluation_guidance_ \\
\hline $\begin{array}{l}\text { 'Barriers and } \\
\text { facilitators' }\end{array}$ & $\begin{array}{l}\text { Aspects related to the individual (ie, healthcare practitioner or healthcare recipient) or to the } \\
\text { organisation that 'determine its degree of readiness to implement, barriers that may impede } \\
\text { implementation, and strengths that can be used in the implementation effort }{ }^{50}\end{array}$ & $\begin{array}{l}\text { Drivers } \\
\text { Mediators, Moderators } \\
\text { Contextual factors } \\
\text { Enablers } \\
\text { Organisational conditions for change }\end{array}$ \\
\hline
\end{tabular}


Table 2 Standards for Reporting Implementation Studies (StaRI): the StaRI checklist

$\begin{array}{ll}\begin{array}{l}\text { Report the } \\ \text { following }\end{array} & \text { 'Implementation strategy' refers to how the intervention was implemented } \\ & \text { 'Intervention' refers to the healthcare or public health intervention that is ben }\end{array}$ 'Intervention' refers to the healthcare or public health intervention that is being implemented

\section{Implementation strategy}

\section{Checklist item}

Title

Abstract

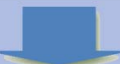

\section{Intervention}

(1)

\begin{tabular}{|c|c|c|c|}
\hline \multirow{2}{*}{ Introduction } & 3 & \multicolumn{2}{|c|}{ Description of the problem, challenge or deficiency in healthcare or public health that the intervention being implemented aims to address } \\
\hline & 4 & $\begin{array}{l}\text { The scientific background and rationale for the implementation strategy } \\
\text { (including any underpinning theory/framework/model, how it is expected to } \\
\text { achieve its effects and any pilot work) }\end{array}$ & $\begin{array}{l}\text { The scientific background and rationale for the intervention being } \\
\text { implemented (including evidence about its effectiveness and how it is } \\
\text { expected to achieve its effects) }\end{array}$ \\
\hline
\end{tabular}

\section{Aims and}

5 The aims of the study, differentiating between implementation objectives and any intervention objectives

objectives

Methods:

description

6 The design and key features of the evaluation (cross-referencing to any appropriate methodology reporting standards), and any changes to study protocol, with reasons

7 The context in which the intervention was implemented (consider social, economic, policy, healthcare, organisational barriers and facilitators that might influence implementation elsewhere)

8 The characteristics of the targeted 'site(s)' (eg, locations/personnel/ resources, etc) for implementation and any eligibility criteria

9 A description of the implementation strategy

The population targeted by the intervention and any eligibility criteria

A description of the intervention

10 Any subgroups recruited for additional research tasks, and/or nested studies are described

Methods: $\quad 11$ Defined prespecified primary and other outcome(s) of the implementation

evaluation strategy, and how they were assessed. Document any predetermined

Defined prespecified primary and other outcome(s) of the intervention targets

(if assessed), and how they were assessed. Document any predetermined targets

12 Process evaluation objectives and outcomes related to the mechanism(s) through which the strategy is expected to work

13 Methods for resource use, costs, economic outcomes and analysis for the Methods for resource use, costs, economic outcomes and analysis for implementation strategy

the intervention

14 Rationale for sample sizes (including sample size calculations, budgetary constraints, practical considerations, data saturation, as appropriate)

15 Methods of analysis (with reasons for that choice)

16 Any a priori subgroup analyses (eg, between different sites in a multicentre study, different clinical or demographic populations), and subgroups recruited to specific nested research tasks 


\section{Table 2 Continued}

\section{Report the 'Implementation strategy' refers to how the intervention was implemented}

following 'Intervention' refers to the healthcare or public health intervention that is being implemented

\section{Implementation strategy}

\section{Intervention}

\section{Checklist item}

Results

17 Proportion recruited and characteristics of the recipient population for the -$$
\text { . }
$implementation strategy

18 Primary and other outcome(s) of the implementation strategy

Proportion recruited and characteristics (if appropriate) of the recipient population for the intervention

Primary and other outcome(s) of the intervention (if assessed)

19 Process data related to the implementation strategy mapped to the mechanism by which the strategy is expected to work

20 Resource use, costs, economic outcomes and analysis for the Resource use, costs, economic outcomes and analysis for the implementation strategy intervention

21 Representativeness and outcomes of subgroups including those recruited to specific research tasks

22 Fidelity to implementation strategy as planned and adaptation to suit Fidelity to delivering the core components of intervention (where context and preferences measured)

23 Contextual changes (if any) which may have affected outcomes

24 All important harms or unintended effects in each group

Discussion 25 Summary of findings, strengths and limitations, comparisons with other studies, conclusions and implications

26 Discussion of policy, practice and/or research implications of the Discussion of policy, practice and/or research implications of the implementation strategy (specifically including scalability) intervention (specifically including sustainability)

General

27 Include statement(s) on regulatory approvals (including, as appropriate, ethical approval, confidential use of routine data, governance approval), trial/ study registration (availability of protocol), funding and conflicts of interest

Note: A key concept is the dual strands of describing, on the one hand, the implementation strategy and, on the other, the clinical, healthcare or public health intervention that is being

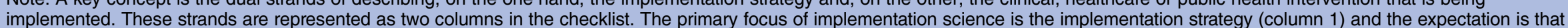
implemented. These strands are represented as two columns in the checklist. The primary focus of implementation science is the implementation strategy (column 1 ) and the expectation is

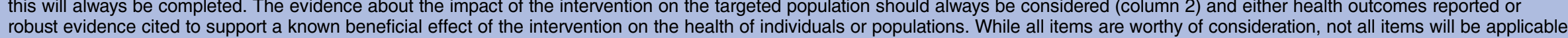
robust evidence cited to support
to or feasible within every study. 
Item 7. Methods: context

The context in which the intervention was implemented. (Consider social, economic, policy, healthcare, organisational barriers and facilitators that might influence implementation elsewhere).

\section{Examples - Context}

The program occurred in three of 14 community-based networks that are part of the statewide Community Care of North Carolina (CCNC) program, an outgrowth of a two-decade effort in North Carolina to better manage the care of Medicaid patients through enhanced patient-centered medical homes. This public-private partnership has five primary components... developed to mirror the components of the Wagner Chronic Care Model for the organization of primary care. At a statewide level, CCNC is operated by North Carolina Community Care Networks, Inc. (NCCCN), a non-profit, tax-exempt organization that facilitates statewide contracting between the 14 CCNC networks and healthcare payers, including Medicaid and Medicare, and allows the participating regional networks to share information technology and other centralized resources. NCCCN also serves as a centralized resource for quality improvement, reporting, web-based case management system, practice support, and provider and member education. ${ }^{22}$

All Italian citizens are covered by a government health insurance and are registered with a general practitioner. Primary care for diabetes is provided by general practitioners and diabetes outpatient clinics. Patients can choose one of these two ways of accessing the healthcare system, according to their preferences, or they can be referred to diabetes outpatient clinics by their general practitioners. The Italian healthcare system includes more than 700 diabetes outpatient clinics. The SINERGIA model is based on a process of disease monitoring and management that tends to exclude the intervention of the diabetologist in the absence of acute problems. Therefore, diabetologists gain time for patients with more severe diabetes, thus enabling them to provide highly qualified care to those patients. ${ }^{35}$

Delivering a multifactorial intervention in our local setting is challenging. Data from a neighboring province showed marked underuse of proven therapies in subjects with diabetes. Furthermore, there is a shortage of physicians, especially in rural areas, while fee-for-service reimbursement may not favor optimal chronic disease management. Although the local prevalence of diabetes (currently 5.3\%) is increasing, the greatest incidence and prevalence are in northern communities, which have the least access to specialists. ${ }^{36}$

\section{Explanation}

Successful implementation of evidence into practice is a planned facilitated process involving the interplay between individuals, evidence and context to promote evidence-informed practice. ${ }^{37} \mathrm{~A}$ rich description of the context is critical to enable readers to assess the external validity of the study, ${ }^{38}$ and decide how the study context compares to their situation and if/how the implementation strategy might be transposed, or need adapting. ${ }^{39}$ Similarly, the social, political and economic context influences the 'entrenched practices and other biases' that hinder evidence-based deimplementation of unproven practices. $^{40} 41$

The Consolidated Framework for Implementation Research (CIFR) defines 39 constructs that may guide reporting of these contextual dimensions (http://www.cfirguide.org/imp.html). The constructs are clustered within five domains: ${ }^{9} 42$

- 'Characteristics of the intervention being implemented' including strength of evidence, adaptability, cost

- 'Outer domain' including alignment with patient needs, peer pressure/competition, external policy, political drivers, economic climate, incentives, timescales

- 'Inner domain' including characteristics and culture of the organisation, perceived need for and capacity to change, leadership and resources

- 'Characteristics of individuals' including attitude, self-efficacy, role within the organisation

- 'Process' by which changes are planned and executed within the organisation

Journal word restrictions will dictate how much detail can be included in the text, but authors should highlight all the key contextual barriers and facilitators that are likely to influence their implementation strategy and outcomes. The examples above highlight the policy context promoting patient-centred medical homes, ${ }^{22}$ the role of diabetologists that enabled a shift in care ${ }^{35}$ and the shortage of specialists that challenged implementation. ${ }^{36}$ Additional information may be provided in an online supplementary file or a separate publication. 
Item 8. Methods: Targeted sites and populations

The characteristics of the targeted 'site(s)' (e.g. locations/ personnel/ resources etc.) for implementation and any

The population targeted by the intervention and any eligibility eligibility criteria.

\section{Examples}

\section{Sites and population targeted by the implementation strategy}

This study comprises nine hospitals in the Netherlands: two academic, four tertiary teaching, and three regional hospitals, with 200 to up to more than 1,300 beds each. ... we believe these hospitals represent the practice of Dutch hospital care. ${ }^{26}$

The study will be implemented in public health facilities in Central and Eastern provinces in Kenya and in three regions in Swaziland... The two criteria for selecting intervention facility selection were: i) good performance in the previous study and ii) high throughput of family planning clients $(\geq 100 /$ month). ${ }^{43}$

\section{Explanation}

Recruitment is considered at two levels:

1. The groups/organisations/locations/providers that were targeted as potential 'sites' for the implementation. Although there may be some overlap with the description of the organisational context (item 7), this is a more specific item related to recruitment strategy including sampling and eligibility criteria. In the second example, the context might describe public health facilities in Kenya and Swaziland; the extract refers to the study-specific requirements of good performance in previous studies and high throughput of family planning clients. ${ }^{43}$ Note that this is a description of targeted sites; a description of participating sites will be in the results.

2. The population targeted by the intervention being implemented including any eligibility criteria. In a clinical context, this might be people with a specific condition (such as requiring abdominal or vascular surgery in the first example), ${ }^{26}$ registered with a participating site, and there may be criteria (such as high risk of mortality ${ }^{26}$ ) that define the population for whom the intervention is appropriate.

Item 9. Methods: Description

A description of the implementation strategy.

\section{Examples}

\section{Description of the implementation strategy}

Implementation planning for this study began with the construction of multiple stakeholder partnerships within the VA PC-MHI program.... [and] was informed by the Promoting Action on Research Implementation in Health Services (PARiHS) framework....

Based on stakeholder feedback and project-team experiences, the implementation strategy for this trial was developed to include three separate but interrelated interventions-online clinician training, clinician audit and feedback, and internal and external facilitation...... emphasis has been placed on understanding stakeholder perspectives, using formative and process evaluations such that the implementation interventions could be modified as needed during the trial. ${ }^{44}$

Sites not initially responding to REP [Replicating Effective Programs] (defined as $<50 \%$ patients receiving $\geq 3$ EBP [evidence-based practice] sessions) will be randomized to receive additional support from an EF or both EF/IF [External/Internal Facilitator]. Additionally, sites randomized to $\mathrm{EF}$ and still not responsive will be randomized to continue with EF alone or to receive EF/IF. The EF provides technical expertise in adapting [Life Goals] in routine practice, whereas the on-site IF has direct reporting relationships to site leadership to support LG use. ${ }^{19}$
A description of the intervention

\section{Description of the intervention}

The ACCESS intervention is a manualized brief CBT [Cognitive Behavioural Therapy] protocol that provides a flexible, patient-centered approach to increase patient engagement and adherence, while addressing both the mental and physical health needs of veterans [chronic obstructive pulmonary disease or heart failure]. ACCESS consists of six weekly treatment sessions and two brief (10- to 15-minute) telephone "booster" sessions within a four month time frame. Participants are asked to attend the first session in person and can participate in subsequent sessions by telephone or in person. ${ }^{44}$

Detailed descriptive information about the content and processes of the ACCESS intervention can be found elsewhere. ${ }^{45}$

The EBP to be implemented is Life Goals (LG) for patients with mood disorders across 80 community-based outpatient clinics... LG is a psychosocial intervention for mood disorders delivered in six individual or group sessions, which includes 10 components: self-management sessions, values, collaborative care, self-monitoring, symptom profile, triggers, cost/benefit analysis of responses, life goals, care management, and provider decision support. Based on social cognitive theory, LG encourages active discussions focused on individuals' personal goals that are aligned with healthy behavior change and symptom management strategies. ${ }^{19}$ 


\section{Explanation}

Descriptions of implementation strategies and complex interventions are criticised as being inconsistently labelled, poorly described, rarely justified, not easy to understand ${ }^{15} 4647$ and not sufficiently detailed to enable the intervention to be replicated. ${ }^{48}$

There needs to be a description of the implementation strategy and the intervention being implemented.

1. Implementation strategies are the 'bundle' of techniques used to enhance the adoption, integration into routine practice and sustainability of a clinical programme or practice. ${ }^{14}$ The Cochrane Effective Practice and Organisation of Care (EPOC) Review Group, considers strategies in the categories of professional, financial, organisational and regulatory. ${ }^{49}$ Others have identified 73 potential implementation techniques from which relevant components may be selected. ${ }^{50} \mathrm{~A}$ framework such as that described by Proctor et $a l^{15}$ would enable consistent comprehensive reporting:

- Actors: The key players (eg, administrators, payers, providers, patients/consumers, advocates) who enact the strategyor enable the strategy to be enacted. The investigator's role should be explicit (eg, a public health strategy over which they have no control; or an implementation process which they are driving).

- Action: The specific activities, steps or processes that constitute the implementation strategy, and how and when these may interact.

- Action target: Strategies may be targeted at specific barriers, enablers, characteristics of the context, processes and other factors influencing the adoption of the intervention. The personnel, organisation or activity targeted by the implementation strategy should be described.

- Temporality: The steps, sequence of actions and timeframe over which the strategy is to be enacted

- Dose: Frequency, duration and intensity of the actions of implementation strategy.

- Implementation outcome(s) likely to be affected by the strategy: Outcomes are defined in item 11; but it may be helpful to signpost these at this stage to ensure that chosen outcomes link explicitly to the implementation strategy and the proposed mechanism of action.

- Theoretical, empirical and/or pragmatic justification for the choice of implementation strategies: These may have been identified in the introduction (item 4), but reference to theoretical models, mapping determinants of practice to effective implementation techniques and any pilot work are likely to be appropriate in the methods.

- Word counts will restrict the description possible within the text, but authors should consider writing a more detailed description, for example, as an online supplementary file. Standards for reporting behavioural change interventions (WIDER) recommend providing access to a manual. ${ }^{51}$ Some have called for an 'intervention bank' in which manuals, videos, descriptions of implementation strategies and interventions, and other related materials can be stored. ${ }^{52}$

- One practical option is to tabulate this information (see table 3). Alternatively, diagrams or schema may be used to represent the interacting components of an implementation strategy. Graphical representations ${ }^{53}$ or 'cascade diagrams' have been devised depicting complex interventions, ${ }^{54}$ and may have potential in describing multilevel implementation studies. Figure 1 is an exemplar using a timeline. ${ }^{18}$

2. The intervention that is being implemented also needs to be described, and any developmental work undertaken to adapt the intervention for implementation cited. ${ }^{55}$ TIDieR highlights the 'who, why, what, where, when and how much' of describing an intervention. ${ }^{33}$ Designed to standardise reporting of the development of complex interventions, the CReDECl checklist ${ }^{56}$ may aid description of developing and piloting implementation of an intervention.

Different sites participating in the implementation study are likely to adapt further the intervention to suit their specific context and authors should consider distinguishing between core components of the intervention (to which fidelity is expected) and elements where adaptation is allowed or even encouraged (see also item 22). A box with details of the intervention may conveniently distinguish it from the description of the implementation strategy. ${ }^{56}$

In study designs that include a comparator group, the description of ' usual care' provided to the nonintervention groups should be sufficiently detailed to enable a reader to judge comparability with their practice and thus the likely impact of the intervention if implemented in their own setting. 
Table 3 Example of a table describing* an implementation strategy compiled from Kilbourne et al ${ }^{19}$ description of the implementation of life goals (LG): a clinical intervention for patients with mood disorders

\begin{tabular}{|c|c|c|c|c|}
\hline $\begin{array}{l}\text { Name of discrete } \\
\text { strategies }\end{array}$ & $\begin{array}{l}\text { Assess for readiness to adopt LG } \\
\text { intervention. Recruit champions } \\
\text { and train for leadership }\end{array}$ & $\begin{array}{l}\text { Develop and distribute } \\
\text { educational materials, manuals, } \\
\text { toolkits and an implementation } \\
\text { blueprint }\end{array}$ & $\begin{array}{l}\text { Educational meetings, outreach } \\
\text { visits, clinical supervision, } \\
\text { technical assistance, ongoing } \\
\text { consultation }\end{array}$ & $\begin{array}{l}\text { Facilitation (external and } \\
\text { internal) and continuous } \\
\text { implementation advice } \\
\end{array}$ \\
\hline Actors & $\begin{array}{l}\text { Investigators, representatives from } \\
\text { practices and community }\end{array}$ & $\begin{array}{l}\text { Investigators, trainers and LG } \\
\text { providers designated at each site }\end{array}$ & Trainers and LG providers. & $\begin{array}{l}\text { Investigators, external and internal } \\
\text { facilitators (EF and IF), LG } \\
\text { providers }\end{array}$ \\
\hline Actions & $\begin{array}{l}\text { Preimplementation meetings with } \\
\text { site representatives for inservice } \\
\text { marketing and dissemination of the } \\
\text { LG programme: overview of LG } \\
\text { evidence, benefits of LG and how to } \\
\text { implement LG. Identify in each site } \\
\text { at least one potential LG providers } \\
\text { with a mental health background and } \\
\text { internal facilitators. Identify } \\
\text { champions. Assess readiness, } \\
\text { barriers and facilitators }\end{array}$ & $\begin{array}{l}\text { Packaging LG protocol and } \\
\text { provider manual (identifying } \\
\text { candidate patients; scripts for } \\
\text { session and follow-up calls; } \\
\text { registry for tracking patients' } \\
\text { progress). Design implementation: } \\
\text { Implementation manual describing } \\
\text { the 'Replicating Effective } \\
\text { Programs' (REP) package. } \\
\text { Patients' workbook (exercises on } \\
\text { behaviour change goals, symptom } \\
\text { assessment, coping strategies...) }\end{array}$ & $\begin{array}{l}\text { Training for } L G \text { providers: evidence } \\
\text { behind LG, core elements and } \\
\text { step-by-step walk through LG } \\
\text { components; patient tracking and } \\
\text { monitoring over time and } \\
\text { continuous education via LG } \\
\text { website. Programme assistance } \\
\text { and LG uptake monitoring via LG } \\
\text { website, support by study } \\
\text { programme assistant, biweekly } \\
\text { monitoring form, feedback reports } \\
\text { and newsletters }\end{array}$ & $\begin{array}{l}\text { Initiation and benchmarking: EF } \\
\text { and LG providers identify barriers, } \\
\text { facilitators, and goals. Leveraging: } \\
\text { IF and LG providers identify } \\
\text { priorities, other LG champions, and } \\
\text { added value of LG to site } \\
\text { providers. Coaching: IF, EF and } \\
\text { LG providers phone to develop } \\
\text { rapport and address barriers. } \\
\text { Ongoing marketing: IF, leaders } \\
\text { and LG providers summarise } \\
\text { progress and develop business } \\
\text { plans }\end{array}$ \\
\hline Targets & $\begin{array}{l}\text { Awareness of evidence-based } \\
\text { interventions, engagement and } \\
\text { settings' readiness to change }\end{array}$ & $\begin{array}{l}\text { Environmental context and } \\
\text { resources, information and access } \\
\text { to interventions }\end{array}$ & $\begin{array}{l}\text { Build knowledge, beliefs, skills and } \\
\text { capabilities: problem solving, } \\
\text { decision-making, interest }\end{array}$ & $\begin{array}{l}\text { Strengths and influences of LG } \\
\text { provider. Measurable objectives } \\
\text { and outcomes in implementing LG }\end{array}$ \\
\hline Temporality & 1st step: preimplementation & 2nd step: REP implementation & 3rd step: training and start up & 4th step: maintenance/evolution \\
\hline Dose & One informative meeting & $\begin{array}{l}\text { For continuous use with every } \\
\text { patient, as needed }\end{array}$ & $\begin{array}{l}\text { 1-day } 8 \text { hour training programme } \\
\text { +continuous assistance and } \\
\text { monitoring }\end{array}$ & $\begin{array}{l}\text { 2-day training programme EF and } \\
\text { continuous facilitation activities }\end{array}$ \\
\hline $\begin{array}{l}\text { Implementation } \\
\text { outcomes } \\
\text { addressed/affected }\end{array}$ & $\begin{array}{l}\text { Barriers, facilitators, specific uptake } \\
\text { goals; organisational factors: ie, } \\
\text { Implementation Leadership Scale, } \\
\text { Implementation Climate Scale, } \\
\text { resources, staff turnover, improved } \\
\text { organisational capacity to implement, } \\
\text { organisational support... }\end{array}$ & $\begin{array}{l}\text { Organisational factors associated } \\
\text { with implementation. Quality of the } \\
\text { supporting materials, packaging } \\
\text { and bundling of the intervention. } \\
\text { Association of available materials' } \\
\text { quality with actual implementation } \\
\text { Providers' knowledge, skills trust }\end{array}$ & $\begin{array}{l}\text { RE-AIM framework (Reach, } \\
\text { Effectiveness, Adoption, } \\
\text { Implementation and Maintenance) } \\
\text { and LG performance measures of } \\
\text { routine clinical care process: ie, } \\
\text { sessions completed by patient, } \\
\text { percentage completing } 6 \\
\text { sessions... }\end{array}$ & $\begin{array}{l}\text { IF, EF and LG provider's } \\
\text { perceptions, strengths and } \\
\text { opportunities to influence site } \\
\text { activities and overcome barriers. } \\
\text { Adaptation and fidelity monitoring: } \\
\text { ie, number of meetings, } \\
\text { opportunities to leverage LG } \\
\text { uptake. Quality and costs }\end{array}$ \\
\hline $\begin{array}{l}\text { Theoretical } \\
\text { justification }\end{array}$ & $\begin{array}{l}\text { CDC's Research to Practice } \\
\text { Framework. Social learning theory }\end{array}$ & \multicolumn{2}{|c|}{$\begin{array}{l}\text { REP framework and implementation strategy for community-based } \\
\text { settings (includes Rogers' Diffusion of Innovations and Social Learning } \\
\text { Theory) }\end{array}$} & $\begin{array}{l}\text { Adaptive implementation.PARiHS } \\
\text { framework }\end{array}$ \\
\hline
\end{tabular}




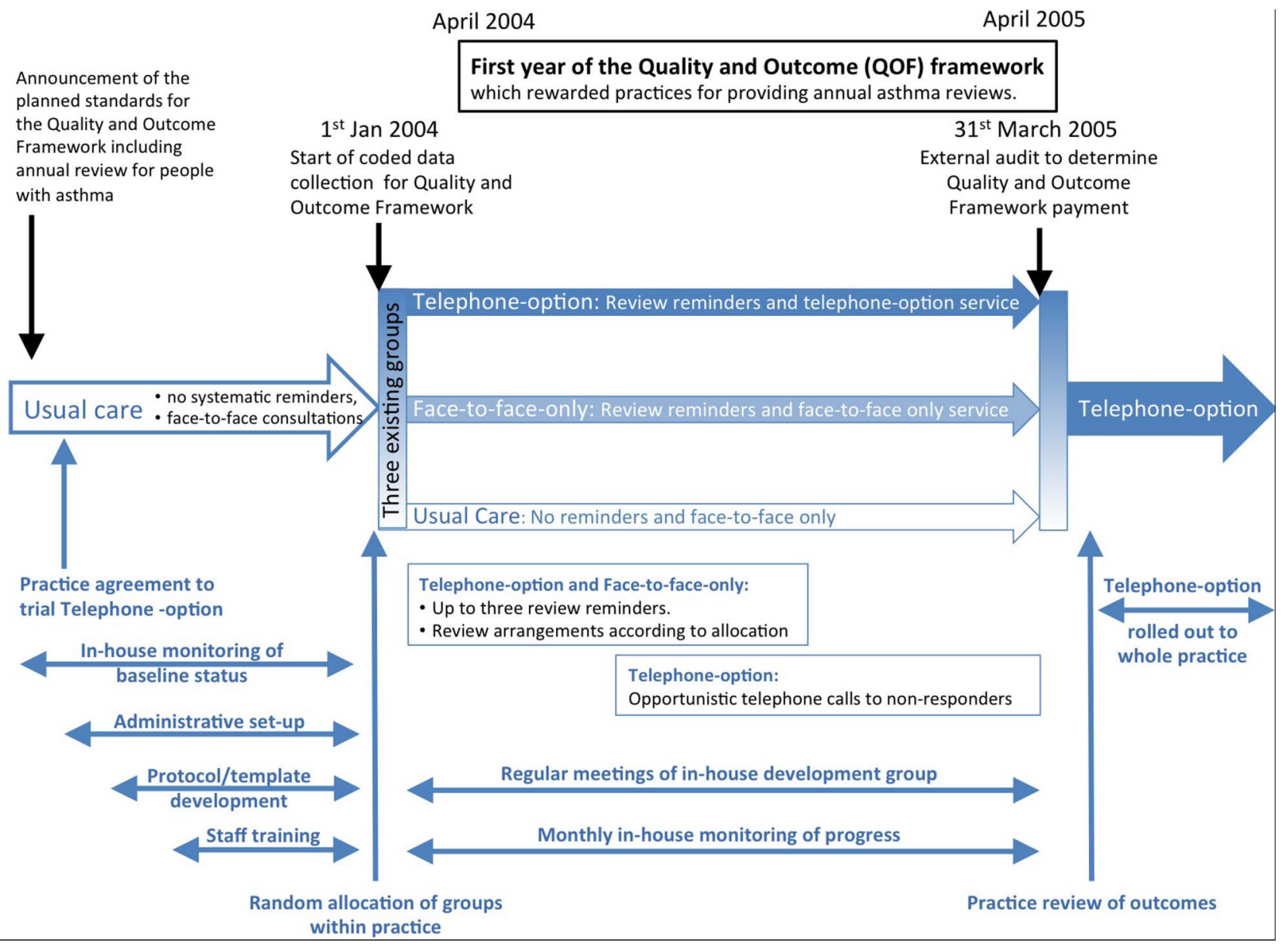

Figure 1 Example of a timeline describing an implementation strategy (compiled from Pinnock et al ${ }^{18}$ description of the implementation of a telephone service for providing asthma reviews). Note: The three-arm implementation study is illustrated in the centre of this schema with the preceding usual care, randomisation on 1 January 2004, the 15-month intervention and subsequent roll-out. The context (specifically the introduction of the Quality and Outcome Framework) is shown at the top of the schema. Below the three-arms of the study are the components of the implementation strategy from set-up and training, ongoing service provision and maintenance and adoption into routine practice.

Item 10. Methods (subgroups)

Any subgroups recruited for additional research tasks, and/or nested studies are described

\section{Examples - Subgroups}

Observations of client-provider interactions: 18 consecutively sampled new family planning/HIV clients and 18 revisit clients ... will be observed. For the postnatal clinic/HIV model ... 24 consecutively sampled postpartum women (within 48 hours of birth, between 1-2 weeks and around 6 weeks postpartum) per study facility (will be recruited) ${ }^{43}$

Researchers posted the following validated questionnaires, with two reminders, to patients with active asthma in the three groups at the end of the study year (excluding children aged $<12$ years, as the questionnaires are not validated for this age group). The only exclusion criteria were a predominant diagnosis of chronic obstructive pulmonary disease, inability to complete the questionnaire (eg, because of severe dementia), and patients excluded by their GP for significant medical or social reasons ${ }^{18}$

\section{Explanation}

Typically in implementation studies, the people targeted by the intervention (eg, patients with a condition registered with a practice or healthcare organisation; population targeted by a public health initiative) will not have consented to the research. Some studies may recruit a subgroup of patients to undertake specific research activities. For example, a proportion of consultations may be observed (see the first example ${ }^{43}$ ), a random sample of patients provided with a new service may be asked to complete questionnaires (see the second example ${ }^{18}$ ) or a purposive sample of stakeholders may be recruited for a qualitative study. The recruitment process for these subgroups should be clearly described. 
Item 11. Methods: Outcomes

Defined pre-specified primary and other outcome(s) of the implementation strategy, and how they were assessed.

Document any pre-determined targets.

\section{Examples}

\section{Outcomes of the implementation strategy}

The primary outcome measure is guideline adherence according to the perioperative Patient Safety Indicators as defined in the national indicator set. This set comprises nine indicators on the processes and structures of care. ${ }^{26}$

Implementation outcomes

To assess brief cognitive behavioral therapy (CBT) adoption and fidelity, as measured by:

a) brief CBT patient engagement (one or more sessions) and adherence (four or more sessions)

b) Department of Veterans Affairs Primary Care-Mental Health Integration [VA PC-MHI] clinician brief CBT adherence and competency ratings as evaluated by expert audio session reviews. ${ }^{44}$
Defined pre-specified primary and other outcome(s) of the intervention (if assessed), and how they were assessed. Document any pre-determined targets.

\section{Outcomes of the intervention}

Secondary (patient) outcomes are in-hospital complications (with particular attention to postoperative wound infections) and hospital mortality, as well as length of hospital stay, unscheduled transfer to the intensive care unit, non-elective hospital readmission, and unplanned reoperation... ${ }^{26}$

Effectiveness outcomes:

To determine whether a brief CBT treatment group as provided by VA PC-MHI clinicians is superior to a usual-care control group at post treatment and 8- and 12-month follow-ups, as measured by:

a) depression and anxiety scores (Patient Health

Questionnaire-9 and Beck Anxiety Inventory)

b) cardiopulmonary disease outcomes (Chronic Respiratory

Questionnaire and Kansas City Cardiomyopathy

Questionnaire). ${ }^{44}$

\section{Explanation}

Figure 2 illustrates the outcomes relevant to implementation science and the StaRI checklist items to which they relate. This schema borrows from the conceptual models and taxonomy of outcomes described by Proctor et al, ${ }^{16} 5758$ but also highlights the dual strands suggested by the StaRI guideline as underpinning reporting implementation intervention studies. The outcomes are mapped to the checklist items in which they are described or reported. The outcomes related to the implementation strategy should be distinguished from outcomes of the intervention:

1. Implementation is the main objective of implementation studies and the primary implementation outcome takes priority.

2. Impact on the primary health outcome is the ultimate aim of implementing the intervention and is therefore important, though it may not always be measured in an implementation study if the underpinning evidence is sufficiently robust (eg, bans to restrict exposure to secondhand smoke ${ }^{59}$ ).

Figure 2 Summary of outcomes and the related items in the Standards for Reporting Implementation Studies checklist.

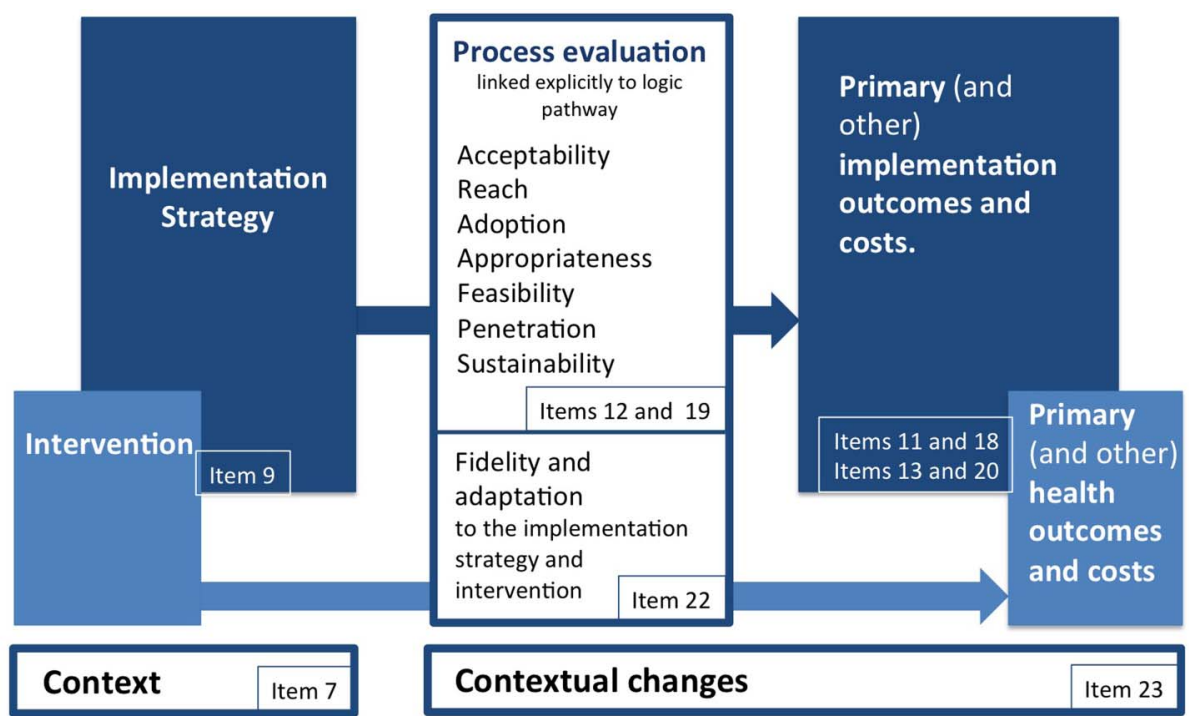

The dual strands are shown as the implementation strategy and implementation outcomes (dark shading) and the intervention and the health outcomes (pale shading). The numbers relate to the checklist where the items are described and reported. 
All outcomes should be clearly defined, including the time point at which they are measured in relation to delivery of the implementation strategy, to enable interpretation of findings in the context of an evolving process of adoption of the intervention within organisations and also inform sustainability.

Not all implementation studies will designate a 'primary outcome', but this is of sufficient importance in the context of experimental designs that the terminology has been retained (see table 2 for alternative terms). This also serves to distinguish implementation outcomes on which a study is powered from the data collected during a process evaluation (see item 12). Feasibility studies may focus on process rather than primary implementation or health outcomes.

The provenance of data is of particular importance in implementation studies in which participants may not be recruited to the research. For example, routine data are typically collected for purposes other than research and the intended use (clinical records, insurance claims, referral patterns, workload monitoring) will influence what and how data are recorded. A description should be provided of the provenance of the data (data source/purpose and process of collection/data completeness) and validity of coding. ${ }^{30}$

It is good practice to define the minimum change that would be considered as representing implementation success (eg, $70 \%$ participation in the intervention) and justify that choice of level.

\section{Item 12. Methods: Process evaluation}

Process evaluation objectives and outcomes related to the mechanism by which the strategy is expected to work.

\section{Examples}

\section{Process evaluation}

The outcomes reported in this paper include adoption, implementation, and maintenance from the Reach, Efficacy/ Effectiveness, Adoption, Implementation, Maintenance (RE-AIM) Model

Adoption was defined as the percentage of clinicians invited to participate who completed training and implemented recommended changes.

Implementation [of the intervention] was determined by how well the practices were able, during each 6-month cycle, to fully incorporate screening and very brief and brief interventions for each behavior into their processes of care, based on information obtained from the chart audits.

Maintenance was determined by the degree to which practices continued to screen for and provide interventions while working on the other behaviors. ${ }^{21}$

In this framework (Hulscher et al. ${ }^{60}$ ), attention is paid to features of the target group, features of the implementers, and the frequency and intensity of intervention activities. Based on this framework we describe the features of the intervention as performed in detail. The process evaluation will furthermore be based on a questionnaire for the contact persons and a questionnaire for the health-care providers to measure their experience with the implementation strategy.." 26

\section{Explanation}

A process evaluation (or formative evaluation) is used to describe the implementation strategy as delivered, and to assess and explore stakeholder experience of the process of implementation and/or target population experiences of receiving the intervention. ${ }^{60-62}$ A process evaluation should be based on an explicit hypothesis (eg, 'logic pathway'; see table 2 for alternative terminology) that spans the mechanism of action of the implementation strategy and the mechanism by which the intervention is expected to improve healthcare. Process data should be related to the hypothesised mechanisms. This implies that data may need to be collected at multiple time points to capture an evolving process, and the relationship between the researcher undertaking the process evaluation and the implementation process (eg, whether interim results are fed back to facilitate adaptation) should be described. ${ }^{61}{ }^{62}$ Context (see item 7) may be reported as a component of the process evaluation. 
For each process evaluation outcome:

- Describe the variables, measures, data sources and data collection methods and frequency, and the analytic approaches employed. Describe who collected data, and the relevance to their role. For example, nurses providing telephone or face-to-face asthma reviews were asked to record duration of consultations, ${ }^{18}$ which may have led to inaccuracies either due to the impracticality of accurate data collection during clinical work and/or bias due to perceived implications for their workload. The provenance of routine data should be described (see item 12).

- Describe methods for assessing fidelity to (and adaptation of) the implementation strategy and to the intervention, sustainability and learning effects (see also item 22). Iterative changes as a result of ongoing feedback should be described.

- Describe checks employed to assess quality of quantitative and/or qualitative data and analysis. For example, nurses' assessment of duration of consultations could be checked against appointment schedules. ${ }^{18}$

Frameworks such as RE-AIM (Reach, Effectiveness, Adoption, Implementation and Maintenance), ${ }^{63}$ diffusion of innovation, ${ }^{64}$ routinisation, ${ }^{65}$ NPT (Normalisation Process Theory), ${ }^{66}$ Framework for process evaluation of cluster-randomised controlled trials $(\mathrm{RCT})^{67}$ or Stages of Implementation Completion, ${ }^{68}$ CIFR (Consolidated Framework for Implementation Research), ${ }^{9} 42$ Theoretical Domains Framework ${ }^{69}$ may be useful in developing, analysing and reporting process evaluations.

Item 13. Methods: Economic evaluation

Methods for resource use, costs, economic outcomes and Methods for resource use, costs, economic outcomes and analysis for the implementation strategy analysis for the intervention

Examples

Economic evaluation of the implementation strategy

Cost analysis of developing and implementing the three interventions from a national perspective (cost of rolling out a particular intervention across the NHS)...20

Financial data were obtained for the costs of setting up and running the Improving Access to Psychological Therapies (IAPT) service for the 2 years of the study, including training, equipment, facilities and overheads, to provide estimates of the costs associated with IAPT. Set-up costs were a small proportion of total costs (less than 10\%) and these were therefore apportioned to this 2-year period rather than the lifetime of the service. ${ }^{70}$

\section{Economic evaluation of the intervention}

... from the perspective of a single trust (cost of all activity and resource used by trust employees in implementation) ${ }^{20}$

The service recorded contact ... time in minutes for each service user and this was used to calculate total contact time over the 2 years, which was combined with total cost data to generate an average cost per minute for the IAPT service... All health and social care services [were] valued using national unit costs.

A broader perspective of costs was taken by assessing productivity impact, which we valued using the lost number of days from work using a human capital approach. ${ }^{70}$

\section{Explanation}

Economic evaluation can inform future implementation and commissioning decisions. Reporting should adhere to existing guidelines relevant to the study design (eg, the International Society for Pharmacoeconomics and Outcomes Research (ISPOR) task force guidelines for economic evaluation (including model-based economic evaluation) ${ }^{71}$ and budget impact analysis, ${ }^{72}$ and guidance on social return on investment approaches ${ }^{73}$ ). This may require an online supplementary file or a separate publication.

An additional requirement in reporting implementation research is to relate economic information to the implementation strategy or the intervention that is being implemented. If possible, reporting should distinguish between the two, with the practicality of doing so ideally having been considered at design stage. A budget impact analysis estimates changes in the expenditure of a healthcare system after adoption of a new intervention, and will be of particular interest to those who plan healthcare budgets. ${ }^{72}$

Reporting should be transparent and cover the following aspects of the evaluation, as relevant:

- Target/eligible population, health system, setting, location and comparator(s).

- Perspective (ie, which resources and costs are being considered) using an equivalent approach for intervention and comparator scenarios, with additional and separate estimates specifically related to the implementation strategy and intervention.

- Time horizon of the evaluation and (if relevant) the discount rate used.

- Methods and sources used to derive resource use and cost estimates.

- Currency, price date and any conversions.

- Outcome/effectiveness measure(s). 
- Statistical approaches for analysis of resource use, costs and outcomes, including handling of joint distributions between these parameters, handling of missing data and any specific considerations, for example, cluster randomisation. ${ }^{74}$

- For models and budget impact analyses, the choice of model/framework, its structure (with graphical representation) and methods for checking consistency and validity.

- For approaches that report composite cost and outcome metrics (eg, incremental cost-effectiveness ratio (ICER) or probability of cost-effectiveness against a given willingness-to-pay threshold), the outcome should be clearly specified and justified (particularly if it is not the same as the primary outcome for the related effectiveness evaluation).

- Assumptions made, and any planned sensitivity/scenario analyses to explore the impact of such assumptions.

\section{Item 14. Methods: sample size}

Rationale for sample sizes (including sample size calculations, budgetary constraints, practical considerations, data saturation, as appropriate)

\section{Example}

\section{Sample size}

Assuming an alpha of 0.05 and a beta of 0.90 , an improvement in perceived daily functioning (defined as a score less than or equal to 4 on the Daily Functioning Thermometer, our primary outcome) at T12 occurring in $20 \%$ of the patients in the intervention group versus $5 \%$ of those in the control group requires at least a net number of 116 patients per arm $(\mathrm{N}=232 ; 5$ patients per practice nurse). It will be necessary to take account of a possible dependence between observations on patients of the same practice nurse (PN). The intra-class correlation coefficient (ICC) is assumed to be 0.04 , a median value for cluster-RCTs in the primary care setting. ${ }^{33}$ Assuming a $30 \%$ loss to follow-up we need to recruit at least 331 patients $(8$ per $\mathrm{PN}$ ). Since participation in the screening procedure will not necessarily mean that patients also give informed consent for the effect evaluation, 10 consecutive patients for each $\mathrm{PN}$ will be invited to participate in the effect evaluation $(\mathrm{N}=460){ }^{24}$

\section{Explanation}

It is important to recruit sufficient participants to be able to address the study's implementation objectives; the rationale for the number of sites and/or people recruited to the study needs to be justified. In a trial (eg, a cluster RCT), this will be based on a sample size calculation using the primary implementation outcome. If health outcomes are also being assessed, consideration may need to be given to the sample size for the primary health outcome. Design-specific advice on reporting sample size calculations can be found in relevant reporting standards. ${ }^{27-29} 3175$ In studies using qualitative methodology, data saturation may inform the final sample size. Budgetary constraints and other pragmatic considerations may also be relevant (such as evaluating an initiative in which size is already determined; in the second example, the sample was 'all active asthmatics' in the practice $^{18}$ ).

\section{Item 15. Methods: analysis}

Methods of analysis (with reasons for that choice)

\section{Example \\ Analysis \\ Numerical data}

Analysis was conducted at the cluster level for each Trust... At each time-point, the differences in mean fasting times between the three intervention groups were compared using analysis of variance (ANOVA). A repeated measure ANOVA across the time-point means for all trusts, within each intervention group, was conducted. The trend coefficient was not significantly different to zero: there was no evidence of trend over time pre-or post-intervention therefore data were combined across timepoints ( 1 to 4 and 5 to 8 ) and simple pre- and post-interventions comparisons were conducted using t-tests. The significance level used for all tests was $5 \%$.

The effect size was calculated for each of the web-based and PDSA interventions compared to standard deviation for change in fluid fasting time between pre- and post-intervention....

Patient experience questionnaires were analysed in SPSS using descriptive statistics, chi squared tests were used to compare characteristics pre- and postintervention.... Descriptive and inferential statistics were conducted (on learning organisational data].

Qualitative data

Audio-recorded individual and focus group interviews were transcribed in full. Data were analysed within data set and managed in N*DIST 5 (pre-intervention) and NVIVO 7 (post-intervention). A combined inductive and deductive thematic analysis process was used....

Synthesis

The theoretical framework [developed for this study is based on the Promoting Action on Research Implementation in Health Services (PARIHS) framework guided the integration of findings across data sets. ${ }^{20}$ 


\section{Explanation}

Design-specific advice on reporting analysis can be found in relevant standards. ${ }^{27-29} 313275$ Consideration needs to be given to the analysis of primary implementation outcomes and then (if measured) to any health outcomes.

In mixed methods studies, clarity is needed about how different data types (numeric, qualitative) will be managed and analysed. ${ }^{76}$ The synthesis of qualitative and quantitative data will be guided by the study's question(s) or objective(s), and by its overarching theoretical framework or theory. Reporting should describe and explain implementation processes (eg, delivery of intervention, facilitators, barriers), contexts (eg, characteristics and influence of) and impacts.

\section{Item 16. Methods: Sub-group analyses}

Any a priori sub-group analyses (e.g. between different sites in a multicentre study, different clinical or demographic populations), and sub-groups recruited to specific nested research tasks

\section{Example - Subgroups}

Planned subgroup analyses focus on subgroups of young women. Age is a core issue in gender violence and HIV incidence. ... A further subgroup analysis will examine the effect of the presence of other programmes for HIV prevention, youth empowerment and reduction in gender violence active in the clusters, with this information collected at the time of the impact survey $^{77}$

\section{Explanation}

Subgroups should be specified a priori and the method of subgroup analysis clearly specified. Further detail on reporting analysis of data from subgroups is available in design-specific reporting standards. ${ }^{27-29} 3175$

\section{Item 17. Results: Populations}

Proportion recruited and characteristics of the recipient population for the implementation strategy.

\section{Examples \\ Characteristics of recipients of the implementation strategy}

Six practices were classed as rural, seven as urban.

Practice list size ranged from 2,300 to 12,500

(median=7,500, IQR 5,250-10,250). Four urban and three rural practices were randomly assigned to the intervention group. $^{78}$

Forty-three practices were randomized: 22 to the intervention group and 21 to control. Massachusetts practices were a mix of hospital clinics, independent community health centers, and private practices. In Michigan, all sites were a part of the Henry Ford Health System; 1 was hospital-based. ... There were no significant differences in practice characteristics between intervention and comparison groups. ${ }^{79}$
Proportion recruited and characteristics (if appropriate) of the recipient population for the intervention

\section{Characteristics of recipients of the intervention}

4,434 adult (age range 18 to 55 years) patients with an asthma diagnosis made more than 12 months previously were identified.... A total of 1572 patients, who had received repeat prescriptions for $\beta 2$-agonists in the previous 12 months, were defined as active asthma patients. Of these, 667 (42\%) were considered to have poorly controlled asthma... ${ }^{78}$

The 43 practices identified a total of 13878 pediatric patients with asthma who may have been eligible for this study. ...Unexpectedly, at baseline, $53 \%$ of the children in the intervention group had a written asthma management plan, compared with $37 \%$ of the children in the control group (P0.001). The groups were not different at baseline with respect to any other measure. ${ }^{79}$

\section{Explanation}

As in cluster RCTs, the populations need to be considered at two levels:

1. Characteristics of the participating sites (eg, demography of a practice/clinic) and the personnel (professional training, staff skills) who were recipients of the implementation strategy, and control groups (if applicable), and their representativeness compared with the sites targeted. Note that characteristics of targeted sites are reported in the methods (item 8)

2. Characteristics of recipients of the health intervention. As these individuals will often not have consented to participate in the research, information is likely to be limited to routine anonymised data.

At each level, reach (the proportion of eligible population who participated and their characteristics) needs to be reported. A diagram illustrating the flow of targeted/participating sites, professionals and patients may be helpful, potentially adapted from CONSORT standards for cluster RCTs. ${ }^{27}$ Published examples of diagrams include a cohort study; ${ }^{80}$ a controlled implementation study ${ }^{18}$ and a before and after study. ${ }^{79}$ 


\section{Item 18. Results: Outcomes}

Primary and other outcome(s) of the implementation strategy.

Examples

Primary (and other) outcomes of the implementation strategy

The Assessment of Chronic Illness Care measurements, obtained at the beginning, midpoint and end of the initiatives, provide evidence of the progressive implementation of the components of the CCM [Chronic Care Model]. These results are described using a spider diagram. ${ }^{81}$

There were 4,550 individuals who met inclusion criteria of which 558 individuals were contacted and received at least a phone contact... On average, individuals received 4.5 phone contacts over the 6-month intervention period. ${ }^{22}$
Primary and other outcome(s) of the Intervention (if assessed)

\section{Primary (and other) outcomes of the intervention (if assessed}

Changes in measures of disease control were more modest.... Nevertheless, tracking aggregate data by means of Shewhart $p$ charts showed special cause variation reflecting improvement in blood pressure and [cholesterol] control in the late stages of the California Collaborative. Significant changes were not seen in HbA1c levels. ${ }^{81}$

During the 90 days prior to the first intervention encounter (index date), $35 \%$ of patients were $>80 \%$ adherent to hypertension medication. By the period of 90-179 days following the first encounter, $54 \%$ had $>80 \%$ adherence for hypertension medication. ${ }^{22}$

\section{Explanation}

We suggest that the primary and other outcomes of the implementation strategy are presented before the impact of the intervention on primary and other health outcomes (if measured). Authors are referred to design-specific standards for detailed advice on reporting outcomes. ${ }^{27-31}$

Item 19. Results: Process evaluation

Process data related to the implementation strategy mapped to the mechanism by which the strategy is expected to work.

\section{Examples}

\section{Process data}

The Park County Diabetes Project made a number of changes in the delivery of diabetes care and patient education. These included establishing and maintaining patient registries; nurses conducting mail and telephone outreach to patients in need of services; mailing personalized patient education materials regarding the $\mathrm{ABCs}$ of diabetes; and providing ongoing continuing education workshops for the health care team. The team redesigned the education curriculum ..., provided group education sessions in community settings, and offered classes regardless of the person's ability to pay. The diabetes nurse in each clinic also provided one-on-one diabetes education.

In October 2000, there were 320 patients with diagnosed diabetes receiving care at these clinics, and that number increased to 392 by February 2003. Among (participating) patients, the proportion receiving an annual foot examination, influenza immunization, and a pneumococcal immunization increased significantly from baseline to follow-up. ${ }^{82}$

We identified three sub-themes that clearly distinguished low from high implementation facilities. First, the high quality of working relationships across service and professional ... boundaries was apparent in the high implementation facilities...... The MOVE! teams at the two high implementation and transition facilities met regularly...... In the low implementation facilities, communications were poor between staff involved with MOVE! and they did much of their communication through email, if at all. ${ }^{83}$

\section{Explanation}

Process evaluation should be related to the logic pathway, capturing the impact of the implementation strategy on intermediate/process outcomes on the pathway, as in the second example in which existing good communication facilitated implementation. It will be important to capture the involvement of the stakeholders in the process of design and implementation (eg, in the first example where the team redesigned the existing education curriculum). Data of importance to the main 'outcome' are likely to include uptake of and attrition from training, implementation tasks, etc, with explanatory insights from qualitative evaluation (eg, in the second example). Contextual changes (see item 23) may be reported as a component of the process evaluation.

If health outcomes are reported, uptake of the intervention by the eligible population will be crucial (as in the first example). Additional papers may be necessary to report all aspects of process data, and to ensure that some publications directly focus on issues of importance to specific groups (eg, policymakers, healthcare managers) ${ }^{61}$ 
Item 20. Results: economic evaluation

Resource use, costs, economic outcomes and analysis for the implementation strategy

\section{Examples}

Health economic outcomes (Implementation strategy)

Estimated total up-front investment for this

Coordinated-Transitional Care (C-TraC) pilot was $\$ 300$ per

person enrolled, which includes all staff, administrative, and implementation costs. ${ }^{84}$

In the base-case analysis, the difference in costs between intervention and control group was $£ 327$, and the difference in QALYs was 0.027 , which generated an ICER point estimate of $£ 12111$ per QALY gained. The probability of the intervention being cost effective was $89 \%$ at the NICE threshold of $£ 30000$ per QALY. ${ }^{85}$
Resource use, costs, economic outcomes and analysis for the intervention

\section{Health economic outcomes (Intervention)}

... given the observed decrease in re-hospitalizations of $5.8 \%$ versus the comparison group, it is estimated that the C-TraC program avoided 361.6 days in acute care over the first 16 months, leading to an estimated gross savings of $\$ 1,202,420$. After accounting for all program costs, this led to estimated net savings of $\$ 826,337$ overall or $\$ 663$ per person enrolled over the first 16 months of the program... 84

\section{Explanation}

Reporting of economic results should adhere to existing relevant guidelines. ${ }^{71} 72$ It should be clear whether the economic results relate to the implementation strategy, the intervention that is being implemented or both. Reporting should be transparent and cover the following aspects of the evaluation:

- Full description of study parameters, including representation of variation, with separate reporting of resource use and costs. For models and budget impact analyses, all input parameters should be reported separately.

- For composite cost and outcome metrics (eg, an ICER or a probability of cost-effectiveness against a given willingness-to-pay threshold), individual costs and outcomes should additionally be reported separately.

- Separate reporting of any sensitivity analyses.

- Provision of budget impact calculators or simulation model programmes may be valued by healthcare decision-makers, and should be developed following specific guidance. ${ }^{72}$

\section{Item 21. Results: subgroups}

Representativeness and outcomes of subgroups including those recruited to specific research tasks

\section{Example}

Representativeness of sub-group

236 (37\% of the 629 patients with poorly-controlled asthma) patients consented to provide questionnaire data. One hundred and six (45\%) patients were from control practices, and 130 (55.1\%) were from intervention practices. Patients with asthma who consented to provide baseline questionnaire data were significantly older, more likely to be female and more affluent than non-consenters. They had significantly fewer $\beta_{2}$ agonists inhaler or courses of oral steroids prescribed in the 12 months pre-study than non-consenters.

One hundred and seventy-seven questionnaires were returned at follow-up out of a possible 236 (75\%). Of these, $78 / 106$ (74\%) were returned by control practice patients and 99/130 (76\%) from intervention practice patients. ${ }^{78}$

\section{Explanation}

Subgroup analyses should be distinguished from outcomes from whole populations (eg, by reporting in a separate table) and their representativeness compared with the whole eligible population. 
Item 22. Results: Fidelity and adaptation

Fidelity to the implementation strategy as planned and adaptation to suit context and preferences.

\section{Examples}

Fidelity and adaptation to the implementation strategy

Although practices were expected to participate fully in the intervention, actual participation varied considerably. Attendance at the 3 learning sessions declined progressively from the first to the third in both states (eg, 34 participants at the first session in Boston; 24 at the third). On average, only $42 \%$ of the practices submitted performance data ... with fewer practices reporting in the later months of the intervention. ${ }^{79}$

At $A D$ [academic detailing] visit $3 \ldots \ldots 46 \%$ of the PDAs [personal digital assistants] indicated that the provider had discontinued use between visits 2 and 3 . ...Several providers reported that, once they adopted electronic medical record systems, they were less inclined to enter data into the PDA (to avoid having to interface with 2 different computers). ${ }^{86}$
Fidelity to delivering the core components of intervention (where measured).

\section{Fidelity and adaptation to the intervention}

[Intervention: adherence to National Cholesterol Education Program clinical practice guidelines] Appropriate management of lipid levels decreased slightly $(73.4 \%$ to $72.3 \%)$ in intervention practices and more markedly $(79.7 \%$ to $68.9 \%)$ in control practices. The net change in appropriate management favored the intervention (+9.7\%; $95 \%$ confidence interval. ${ }^{86}$

\section{Explanation}

Fidelity may be considered at two levels: implementation fidelity and intervention fidelity. Implementation fidelity refers to the degree of adherence to the described implementation strategy. Intervention fidelity is the degree to which an intervention is implemented as prescribed in the original protocol. The implementation strategy and the intervention, however, may need to be adapted if they are to fit within the routines of local practice. ${ }^{86}$ Adaptation is the degree to which the strategy and intervention are modified by users during implementation to suit local needs. ${ }^{26}$ Insufficient fidelity to the 'active ingredients' of an intervention dilutes effectiveness, ${ }^{87}$ whereas insufficient adaptation stifles tailoring potentially diluting effective implementation. ${ }^{88}$ An approach to reporting these apparently contradictory concepts is to define the core components of an intervention to which fidelity is expected, and those aspects which may be adapted by local sites to aid implementation. ${ }^{59} 87$ Distinction may be made between an active process of innovative adaptation that facilitates implementation, passive 'drift' in which tasks are allowed to lapse, ${ }^{89}$ and active subversion which blocks implementation. ${ }^{90}$

Fidelity should be reported:

1. To the core components of the implementation strategy and any adaptations made by participating sites. A systematic meta-review of the literature on fidelity measures described four aspects of fidelity required for a comprehensive assessment (design, training, monitoring of intervention delivery and intervention receipt). ${ }^{91}$

2. To delivery of the core components of the intervention (or at least considered if not measured) and any adaptations made.

Item 23. Results: context

Contextual changes (if any) which may have affected the outcomes

\section{Example}

\section{Contextual changes}

The present study coincided with the introduction of the UK General Medical Services contract in January 2004 which rewards practices who achieve clinical standards, including a target of $70 \%$ for the annual review of people with 'active' asthma. The impact of this was seen in the usual-care group which increased the review rate by $14 \%$ without a structured recall service. ${ }^{18}$

\section{Explanation}

There should be a description of any important contextual changes (or not) occurring during the study that may have affected the impact of the implementation strategy-for example, policy incentives, parallel programmes, changes in personnel, media publicity. The CIFR constructs (see item 7) is a useful framework for describing context, ${ }^{42}$ and a timeline (see item 9 ) may be a convenient way to illustrate potential impact of contextual changes. Contextual changes (see item 19) may be reported as a component of the process evaluation. 
Item 24. Results: harms

All important harms or unintended effects in each group.

\section{Example}

\section{Reporting of harms}

[In the context of a computerised decision support to improve prescribing in pregnancy] Two factors contributed to alerts being based on incorrect patient pregnancy status: either the updated diagnosis had not been coded into administrative data at all or transfer of the updated coded diagnosis information from hospital administrative data to health plan administrative data was delayed. $^{92}$

\section{Explanation}

Adverse or unintended consequences of implementation studies are often under-reported. ${ }^{93-95}$ Any important harms or unintended effects should be reported, quantified (eg, on health outcomes, organisational efficiency or user satisfaction) and possible reasons identified (eg, flaws in the intervention, context challenging implementation). ${ }^{91}$

Item 25: Discussion: summary

Summary of findings, strengths and limitations, comparisons with other studies, conclusions and implications.

\section{Example}

\section{Summary findings}

The participating practices adopted most elements of the CCM [Chronic Care Model], including development of inter-professional teams, delegation of provision of care by appropriate team members, implementation of patient self-management strategies, group visits, proactive patient management-anticipating the needs of patients as opposed to providing reactive management-and use of an information system to track individual patient measures. In addition, resident training programs successfully incorporated educational strategies for learning the elements of evidence-based chronic illness care. $^{81}$

\section{Explanation}

The structure of the discussion will follow the style of the journal, but ideally should include summary of findings, strengths and limitations, comparisons with other studies, implications (see item 26) and conclusions. ${ }^{96}$

\section{Item 26. Discussion: Implications}

Discussion of policy, practice and/or research implications of the implementation strategy (specifically including scalability).

Discussion of policy, practice and/or research implications of the intervention (specifically including sustainability).

\section{Examples}

Implications related to the implementation strategy

These initiatives suggest that both the practice redesign required for implementation of the CCM [Chronic Care Model] and linked educational strategies are achievable in resident continuity practices....

Durable implementation of the CCM in resident practices necessitates substantial commitment from local institutional, clinical and academic leadership. ${ }^{81}$

Including a telephone option as part of a review service for people with asthma is a practical and cost-effective strategy for enhancing access... These findings have direct clinical implications and also policy implications for those setting standards for the Quality and Outcomes Framework of the UK GMS [General Medical Services] contract. $^{18}$

\section{Explanation}

The authors should reflect on:

1. The implications of the success (or otherwise) of the implementation strategy, for research and practice.

2. The health benefits (or otherwise) of implementing the intervention.

Targets might include citizens, practitioners interested in the health intervention, researchers interested in the conceptual and theoretical perspective, managers and clinical leaders interested in implementing the intervention and those interested in the broader policy implications. A key point for consideration will be 'sustainability' (the extent to which the intervention can continue to deliver its intended benefits over an extended period of time after any support has terminated) and how policy could be modified to support ongoing implementation.

Scalability, generalisability, applicability and transferability of the implementation strategy may need to be discussed. 
Item 27. General

Include statement(s) on regulatory approvals (including, as appropriate, ethical approval, confidential use of routine data, governance approval), trial/study registration (availability of protocol), funding and conflicts of interest.

\section{Explanation}

Ethical considerations, regulatory approvals, funding and conflicts of interest (including commercial interests, involvement of the owner of a product in the implementation or evaluation) should be reported. Registration of trials is a requirement, and increasingly recommended for other study designs. ${ }^{29} 76$ While not yet routine practice in implementation studies, authors may find it useful to be able to refer to a published protocol (eg, 19264377 ). The detailed descriptions required to describe context, implementation strategy and the intervention comprehensively will be challenging within the word count of a journal and a published protocol may provide further detail.

\section{CONCLUSIONS}

The StaRI standards are registered with the EQUATOR Network (http://www.equator-network.org) and the checklist is available from this journal and BMJ.com. We invite editors of journals publishing interventional implementation studies to consider requiring submission of a StaRI checklist, and authors reporting such studies to adopt the checklist. We have presented the concepts and sought feedback from delegates at workshops, conference discussions and implementation project steering groups; in general, the concepts seem to resonate with colleagues. In the future, we would like to work with authors as they apply the checklist to their papers, 'road testing' the standards and enabling iterative development.

We are particularly interested in whether the concept of the dual strands (implementation strategy/intervention) resonates with authors and readers of implementation studies. Is it practical to expect authors to consult other methodological checklists for reporting designspecific aspects of their study? We look forward to learning about innovative solutions to providing adequate descriptions of context, implementation strategies and interventions that accommodate the requirements of journals, needs of authors as well as preferences of readers.

Previously published statements have been instrumental in improving reporting standards, ${ }^{10}$ and our hope is that StaRI will achieve a similar improvement in the reporting of implementation strategies that will facilitate translation of effective interventions into routine practice.

\section{Author affiliations}

${ }^{1}$ Asthma UK Centre for Applied Research, Usher Institute of Population Health Sciences and Informatics, The University of Edinburgh, Edinburgh, UK

${ }^{2}$ Research Institute, Hospital for Sick Children, Toronto, Ontario, Canada

${ }^{3}$ Department of Psychiatry and Dalla Lana School of Public Health, University of Toronto, Toronto, Ontario, Canada

${ }^{4}$ Division of Emergency Medicine, Washington University School of Medicine in St Louis, St Louis, USA

${ }^{5}$ Pragmatic Clinical Trials Unit, Centre for Primary Care and Public Health, Blizard Institute, Barts and The London School of Medicine and Dentistry, Queen Mary University of London, London, UK

${ }^{6}$ Primary Care Research Unit of Bizkaia, Basque Health Service, Bilbao, Spain

${ }^{7}$ Centre for Primary Care and Public Health, Blizard Institute, Barts and The London School of Medicine and Dentistry, Queen Mary University of London, London, UK
${ }^{8}$ Bangor Institute for Health and Medical Research, Bangor University, Bangor UK

${ }^{9}$ Montefiore Medical Center, The University Hospital for Albert Einstein College of Medicine, New York, USA

${ }^{10}$ Department of Primary Care and Population Health, University College London, London, UK

Acknowledgements Members of the PRISMS team (Eleni Epiphaniou, Gemma Pearce and Hannah Parke) supported the underpinning literature work, and the e-Delphi was handled by ClinVivo. The authors are grateful to a colleagues (implementation science experts, healthcare researchers, clinicians, $\mathrm{PhD}$ students) who reviewed the penultimate draft of the StaRI statement and provided a reality check and constructive feedback: Helen Ashdown, David Chambers, Louise Craig, Clarisse Dibao-Dina, Peter Hanlon, Roger Jones, Rachel Jordan, Chris del Mar, Brian McKinstry, Susan Morrow, John Ovretveit, David Price, Kamran Siddiqui, Rafael Stelmach, Paul Stephenson, Shaun Treweek, Bryan Weiner. The authors also thank Melissa Goodbourn and Allison Worth who arranged feedback from the Edinburgh Clinical Research Facility Patient Advisory Panel (Stephanie Ashby, Alison Williams) and Steven Towndrow who coordinated feedback from the Patient and Public Involvement representatives of the NIHR CLAHRC North Thames (Ben Wills-Eve, Rahila Bashir, Julian Ashton, Colleen Ewart, Karen Williams).

Collaborators The StaRI Group includes: MB, CRC, Peter Craig, SE, Eleni Epiphaniou, GG, JR-M, PM, Brian Mittman, EM, AP, Gemma Pearce, HP, AS and SJCT.

Contributors HP initiated the idea for the study and with SJCT led the development of the protocol, securing of funding, study administration, workshop and writing of the paper. AS, CJG and SE advised on the development of the protocol, and data analysis. All authors participated in the StaRI international working group along with GP, BM and MG. HP wrote the initial draft of the paper, to which all the authors contributed. HP is the study guarantor.

Funding The StaRI initiative and workshop was funded by contributions from the Asthma UK Centre for Applied Research (AC-2012-01); Chief Scientist Office, Scottish Government Health and Social Care Directorates

(PCRCA_08_01); the Centre for Primary Care and Public Health, Queen Mary University of London; and with contributions in kind from the PRISMS team (NIHR HS\&DR Grant ref: 11/1014/04). SJCT was (in part) supported by the National Institute for Health Research (NIHR) Collaboration for Leadership in Applied Health Research and Care (CLAHRC) North Thames at Bart's Health NHS Trust. AS is supported by the Farr Institute.

Disclaimer The funding bodies had no role in the design, in the collection, analysis, and interpretation of data; in the writing of the manuscript; nor in the decision to submit the manuscript for publication. The views expressed are those of the author(s) and not necessarily those of the NHS, the NIHR or the Department of Health.

Competing interests All authors have completed the ICMJE uniform disclosure form at http://www.icmje.org/coi_disclosure.pdf and declare: research grants from Chief Scientist Office (HP), Asthma UK (AS, HP, SJCT), Farr Institute (AS), NIHR HS\&DR (HP, SJCT), NIHR CLAHRC (SJCT) for the submitted work; no financial relationships with any organisations that might have an interest in the submitted work in the previous 3 years; CRC is the 
Deputy Editor-in-Chief for Academic Emergency Medicine and on the editorial boards for the Journal of the American Geriatrics Society and Annals of Internal Medicine's ACP Journal Club and serves as paid faculty for Emergency Medical Abstracts, JR-M is the Director of the NIHR HS\&DR Programme, no other relationships or activities that could appear to have influenced the submitted work.

Provenance and peer review Not commissioned; externally peer reviewed.

Data sharing statement No additional data are available.

Open Access This is an Open Access article distributed in accordance with the Creative Commons Attribution Non Commercial (CC BY-NC 4.0) license, which permits others to distribute, remix, adapt, build upon this work noncommercially, and license their derivative works on different terms, provided the original work is properly cited and the use is non-commercial. See: http:// creativecommons.org/licenses/by-nc/4.0/

\section{REFERENCES}

1. Harvard Catalyst. Clinical and Translational Research Spectrum. https://catalyst.harvard.edu/pathfinder (accessed Jun 2016).

2. Pinnock H, Epiphaniou E, Pearce G, et al. Implementing supported self-management for asthma: a systematic review of implementation studies. BMC Med 2015;13:127.

3. Rycroft-Malone J, Burton CR. Is it time for standards for reporting on research about implementation? Worldviews Evid Based Nurs 2011;8:189-90.

4. Chalkidou K, Anderson G. Comparative Effectiveness Research: International Experiences and Implications for the United States. http://www.nihcm.org/pdf/CER_International_Experience_09.pdf (accessed Jun 2016).

5. Pinnock H, Barwick M, Carpenter C, et al., for the StaRI team. Standards for Reporting Implementation Studies (StaRI) statement. BMJ 2017;356:i6795.

6. Moher D, Schulz KF, Simera I, et al. Guidance for developers of health research reporting guidelines. PLoS Med 2010;7:e1000217.

7. Pinnock H, Taylor S, Epiphaniou E, et al. Developing Standards for Reporting Phase IV Implementation studies. http://www. equator-network.org/wp-content/uploads/2013/09/ Proposal-for-reporting-guidelines-of-Implementation-Research-StaRI pdf (accessed Jun 2016)

8. Pinnock H, Epiphaniou E, Sheikh A, et al. Developing Standards for Reporting Implementation studies (StaRI): an e-Delphi. Implement Sci 2015;10:42

9. Damschroder LJ, Aron DC, Keith RE, et al. Fostering implementation of health services research findings into practice: a consolidated framework for advancing implementation science. Implement Sci 2009;4:50.

10. Foy R, Sales A, Wensing M, et al. Implementation science: a reappraisal of our journal mission and scope. Implement Sci 2015;10:51

11. Eccles MP, Armstrong D, Baker R, et al. An implementation research agenda. Implement Sci 2009;4:18.

12. Brownson RC, Colditz GA, Proctor EK, eds. Dissemination and implementation research in health: translating science into practice. New York: Oxford University Press, 2012.

13. Craig $\mathrm{P}$, Dieppe $\mathrm{P}, \mathrm{Macintyre} \mathrm{S}$, et al. Developing and evaluating complex interventions: the new Medical Research Council guidance. London: MRC, 2008. http://www.mrc.ac.uk/ complexinterventionsguidance (accessed Jun 2016).

14. Curran GM, Bauer M, Mittman B, et al. Effectiveness-implementation hybrid designs: combining elements of clinical effectiveness and implementation research to enhance public health impact. Med Care 2012:50:217-26.

15. Proctor EK, Powell BJ, McMillen JC. Implementation strategies: recommendations for specifying and reporting. Implement Sci 2013;8:1-11

16. Rabin BA, Brownson RC. Developing the terminology for dissemination and implementation research. In: Brownson RC, Colditz GA, Proctor EK, eds. Dissemination and implementation research in health. New York: Oxford University Press, 2012, pp 2352.

17. Proctor E, Brownson RC. Measurement issues in dissemination and implementation research. In: Brownson RC, Colditz GA, Proctor EK, eds. Dissemination and implementation research in health. New York: Oxford University Press, 2012, pp 261-80.

18. Pinnock H, Adlem L, Gaskin S, et al. Accessibility, clinical effectiveness and practice costs of providing a telephone option for routine asthma reviews: phase IV controlled implementation study. Br J Gen Pract 2007:57:714-22.

19. Kilbourne AM, Almirall D, Eisenberg D, et al. Protocol: Adaptive Implementation of Effective Programs Trial (ADEPT): cluster randomized SMART trial comparing a standard versus enhanced implementation strategy to improve outcomes of a mood disorders program. Implement Sci 2014:9:132.

20. Rycroft-Malone J, Seers K, Crichton N, et al. A pragmatic cluster randomised trial evaluating three implementation interventions. Implement Sci 2012;7:80

21. Aspy CB, Mold JW, Thompson DM, et al. Integrating screening and interventions for unhealthy behaviors into primary care practices. $A m$ J Prev Med 2008;35:S373-80.

22. Bosworth HB, DuBard A, Ruppenkamp J, et al. Evaluation of a self-management implementation intervention to improve hypertension control among patients in Medicaid. Transl Behav Med 2011;1:191-9.

23. Guyatt $\mathrm{GH}$, Oxman $\mathrm{AD}$, Schünemann $\mathrm{HJ}$, et al. GRADE guidelines: a new series of articles in the Journal of Clinical Epidemiology. J Clin Epidemiol 2010;64:380-2.

24. van Dijk-de Vries A, van Bokhoven MA, Terluin B, et al. Integrating nurse-led Self-Management Support (SMS) in routine primary care: design of a hybrid effectiveness-implementation study among type 2 diabetes patients with problems of daily functioning and emotional distress: a study protocol. BMC Fam Pract 2013;14:77.

25. Sämann A, Lehmann T, Kloos $C$, et al. Flexible, intensive insulin therapy and dietary freedom in adolescents and young adults with type 1 diabetes: a prospective implementation study. Diabet Med 2008:25:592-6.

26. Emond YE, Calsbeek H, Teerenstra S, et al. Improving the implementation of perioperative safety guidelines using a multifaceted intervention approach: protocol of the IMPROVE study, a stepped wedge cluster randomized trial. Implement Sci 2015;10:3.

27. Campbell MK, Piaggio G, Elbourne DR, et al., for the CONSORT Group. Consort 2010 statement: extension to cluster randomised trials. BMJ 2012;345:e5661.

28. Zwarenstein M, Treweek S, Gagnier JJ, et al., for the CONSORT and Pragmatic Trials in Healthcare (Practihc) Groups. Improving the reporting of pragmatic trials: an extension of the CONSORT statement. BMJ 2008;337:a2390.

29. von Elm E, Altman DG, Egger M, et al., for the STROBE Initiative. The Strengthening the Reporting of Observational Studies in Epidemiology (STROBE) statement: guidelines for reporting observational studies. J Clin Epidemiol 2008;61:344-9.

30. Nicholls SG, Quach P, von Elm E, et al. The REporting of studies Conducted using Observational Routinely-collected health Data (RECORD) Statement: methods for arriving at consensus and developing reporting guidelines. PLOS ONE 2015;10:e0125620.

31. Jarlais DCD, Lyles C, Crepaz N. Improving the reporting quality of nonrandomized evaluations of behavioral and public health interventions: the TREND statement. Am J Public Health 2004;94:361-6.

32. Tong A, Sainsbury P, Craig J. Consolidated criteria for reporting qualitative research (COREQ): a 32-item checklist for interviews and focus groups. Int J Qual Health Care 2007;19:349-57.

33. Hoffmann TC, Glasziou PP, Boutron I, et al. Better reporting of interventions: template for intervention description and replication (TIDieR) checklist and guide. BMJ 2014;348:g1687.

34. Davidoff $F$, Batalden $P$, Stevens $D$, et al. Publication guidelines for quality improvement studies in health care: evolution of the SQUIRE project. Qual Saf Health Care 2008;17:i3-9.

35. Musacchio N, Lovagnini Scher A, Giancaterini A, et al. Impact of a chronic care model based on patient empowerment on the management of Type 2 diabetes: effects of the SINERGIA programme. Diabet Med 2011;28:724-30.

36. Senior PA, MacNair L, Jindal K. Delivery of multifactorial interventions by nurse and dietitian teams in a community setting to prevent diabetic complications: a quality-improvement report. $\mathrm{Am}$ $J$ Kidney Dis 2008:51:425-34.

37. Rycroft-Malone J, Seers K, Chandler J, et al. The role of evidence, context, and facilitation in an implementation trial: implications for the development of the PARIHS framework. Implement Sci 2013;8:28.

38. Green LW, Glasgow RE. Evaluating the relevance, generalization, and applicability of research: issues in external validation and translation methodology. Eval Health Prof 2006;29:126-53.

39. Edward N, Barker PM. The importance of context in implementation research. J Acquir Immune Defic Syndr 2014;67(Suppl 2): S157-62.

40. Prasad V, Ioannidis JPA. Evidence-based de-implementation for contradicted, unproven, and aspiring healthcare practices. Implement Sci 2014;9:1. 
41. Montini T, Graham ID. "Entrenched practices and other biases": unpacking the historical, economic, professional, and social resistance to de-implementation. Implement Sci 2015;10:24.

42. Consolidated Framework for Implementation Research. CFIR Constructs. http://cfirguide.org/index.html (accessed Jun 2016).

43. Warren CE, Mayhew SH, Vassall A, et al. Study protocol for the Integra Initiative to assess the benefits and costs of integrating sexual and reproductive health and HIV services in Kenya and Swaziland. BMC Public Health 2012;12:973.

44. Cully JA, Armento MEA, Mott J, et al. Brief cognitive behavioral therapy in primary care: a hybrid type 2 patient-randomized effectiveness-implementation design. Implement Sci 2012;7:64.

45. Cully JA, Paukert A, Falco J, et al. Cognitive-behavioral therapy: innovations for cardiopulmonary patients with depression and anxiety. Cogn Behav Pract 2009;16:394-407.

46. Michie S, Fixsen DL, Grimshaw JM, et al. Specifying and reporting complex behaviour change interventions: the need for a scientific method. Implement Sci 2009;4:1-6.

47. Davies P, Walker AE, Grimshaw JM. A systematic review of the use of theory in the design of guideline dissemination and implementation strategies and interpretation of the results of rigorous evaluations. Implement Sci 2010;5:1-6.

48. Hoffmann TC, Erueti C, Glasziou PP. Poor description of non-pharmacological interventions: analysis of consecutive sample of randomised trials. BMJ 2013;347:f3755.

49. Cochrane Effective Practice and Organisation of Care Review Group. Data Collection Checklist. 2002. http://epoc.cochrane.org/ sites/epoc.cochrane.org/files/uploads/datacollectionchecklist.pdf (accessed Jun 2016)

50. Powell BJ, Waltz TJ, Chinman MJ, et al. A refined compilation of implementation strategies: results from the Expert Recommendations for Implementing Change (ERIC) project. Implement Sci 2015;10:21.

51. Albrecht L, Archibald M, Arseneau D, et al. Development of a checklist to assess the quality of reporting of knowledge translation interventions using the Workgroup for Intervention Development and Evaluation Research (WIDER) recommendations. Implement Sci 2013;8:52.

52. Glaziou P, Chalmers I, Altman DG, et al. Taking healthcare interventions from trial to practice. BMJ 2010;341:c3852.

53. Perara R, Heneghan C, Yudkin P. Graphical method for depicting randomized trials of complex interventions. BMJ 2007;334:127-9.

54. Hooper R, Froud RJ, Bremner SA, et al. Cascade diagrams for depicting complex interventions in randomised trials. BMJ 2013;347: f6681.

55. Ogrinc G, Davies L, Goodman D, et al. SQUIRE 2.0 (Standards for QUality Improvement Reporting Excellence): revised publication guidelines from a detailed consensus process. BMJ Qual Saf 2015;46:501-7.

56. Möhler R, Bartoszek G, Köpke S, et al. Proposed criteria for reporting the development and evaluation of complex interventions in healthcare (CReDECI): guideline development. Int J Nurs Stud 2012;49:40-6.

57. Proctor EK, Landsverk J, Aarons G, et al. Implementation research in mental health services: an emerging science with conceptual, methodological, and training challenges. Adm Policy Ment Health 2009;36:24-34.

58. Proctor $\mathrm{E}$, Silmere $\mathrm{H}$, Raghavan $\mathrm{R}$, et al. Outcomes for implementation research: conceptual distinctions, measurement challenges, and research agenda. Adm Policy Ment Health 2011;38:65-76.

59. Been JV, Nurmatov UB, Cox B, et al. Effect of smoke-free legislation on perinatal and child health: a systematic review and meta-analysis. Lancet 2014;383:1549-60.

60. Hulscher M, Laurant M, Grol R. Process evaluation of implementation strategies. In: Grol R, Wensing M, Eccles M, et al, eds. Improving patient care: the implementation of change in health care. 2nd edn. Oxford: John Wiley \& Sons Ltd, pp 256-72.

61. Moore G, Audrey S, Barker M, et al., for the MRC Population Health Science Research Network. Process evaluation of complex interventions. UK Medical Research Council (MRC) guidance. BMJ 2015;350:h1258.

62. Stetler C, Legro MW, Wallace CM, et al. The role of formative evaluation in implementation research and the QUERI experience. $J$ Gen Intern Med 2006;21(Suppl 2):S1-8.

63. Glasgow RE, Vogt TM, Boles SM. Evaluating the public health impact of health promotion interventions: the RE-AIM framework. Am $J$ Public Health 1999;89:1922-7.

64. Greenhalgh T, Robert G, Macfarlane F, et al. Diffusion of innovations in service organizations: systematic review and recommendations. Milbank Q 2004;82:581-629.

65. Greenhalgh T. Role of routines in collaborative work in healthcare organisations. BMJ 2008;337:a2448.
66. May C. A rational model for assessing and evaluating complex interventions in health care. BMC Health Serv Res 2006;6:86.

67. Grant A, Treweek S, Dreischulte T, et al. Process evaluations for cluster-randomised trials of complex interventions: a proposed framework for design and reporting. Trials 2013;14:15.

68. Saldana $\mathrm{L}$. The stages of implementation completion for evidence-based practice: protocol for a mixed methods study. Implement Sci 2014;9:43.

69. Michie S, Johnston M, Abraham C, et al., on behalf of the 'Psychological Theory' Group. Making psychological theory useful for implementing evidence-based practice; a consensus approach. Qual Saf Health Care 2005;14:26-33.

70. Mukuria C, Brazier J, Barkham M, et al. Cost-effectiveness of an improving access to psychological therapies service. Br J Psychiatry 2013;202:220-7.

71. Husereau D, Drummond M, Petrou S, et al., on behalf of the CHEERS Task Force. Consolidated Health Economic Evaluation Reporting Standards (CHEERS) statement. BMJ 2013;346:f1049.

72. Sullivan SD, Mauskopf JA, Augustovski F, et al. Budget impact analysis-principles of good practice: report of the ISPOR 2012 Budget Impact Analysis Good Practice II Task Force. Value Health 2014;17:5-14

73. Cabinet Office. A guide to social return on investment 2012. https:// www.bond.org.uk/data/files/Cabinet_office_A_guide_to_Social_ Return_on_Investment.pdf (accessed Jun 2016).

74. Gomes M, Grieve R, Nixon R, et al. Statistical methods for cost-effectiveness analyses that use data from cluster randomized trials: a systematic review and checklist for critical appraisal. Med Decis Making 2012;32:209-20.

75. Begg C, Cho M, Eastwood S, et al. Improving the quality of reporting of randomized controlled trials. The CONSORT statement. JAMA 1996;276:637-9.

76. Cresswell JW, Plano-Clark VL. Designing and conducting mixed methods research 2011. CA: Sage Publications, 2011.

77. Andersson N, Cockcroft A, Thabane L, et al. HIV prevention in favour of the choice-disabled in southern Africa: study protocol for a randomized controlled trial. Trials 2013;14:274.

78. Cleland JA, Hall S, Price D, et al. An exploratory, pragmatic, cluster randomised trial of practice nurse training in the use if asthma action plans. Prim Care Respir J 2007;16:311-18.

79. Homer CJ, Forbes P, Horvitz L, et al. Impact of a quality improvement program on care and outcomes for children with asthma. Arch Pediatr Adolesc Med 2005;159:464-9.

80. Jordan RE, Lancashire RJ, Adab P. An evaluation of Birmingham Own Health telephone care management service among patients with poorly controlled diabetes. A retrospective comparison with the General Practice Research Database. BMC Public Health 2011;11:707.

81. Stevens DP, Bowen JL, Johnson JK, et al. A multi-institutional quality improvement initiative to transform education for chronic illness care in resident continuity practices. J Gen Intern Med 2010;25(Suppl 4):S574-80.

82. Dettori N, Flook BN, Pessl E, et al. Improvements in care and reduced self-management barriers among rural patients with diabetes. J Rural Health 2005;21:172-7.

83. Damschroder LJ, Lowery JC. Evaluation of a large-scale weight management program using the consolidated framework for implementation research (CFIR). Implement Sci 2013;8:51.

84. Kind AJH, Brenny-Fitzpatrick M, Leahy-Gross K, et al. Harnessing protocolized adaptation in dissemination: successful implementation and sustainment of the veterans affairs coordinated-transitional care program in a non-veterans affairs hospital. J Am Geriatr Soc 2016;64:409-16.

85. Murphy SM, Edwards RT, Williams N, et al. An evaluation of the effectiveness and cost effectiveness of the National Exercise Referral Scheme in Wales, UK: a randomised controlled trial of a public health policy initiative. J Epidemiol Community Health 2012;66:745-53.

86. Bertoni AG, Bonds DE, Chen $\mathrm{H}$, et al. Impact of a multifaceted intervention on cholesterol management in primary care practices: guideline adherence for heart health randomized trial. Arch Intern Med 2009:169:678-86.

87. Hawe P, Shiell A, Riley T. Complex interventions: how "out of control" can a randomised controlled trial be? BMJ 2004:328:1561-3.

88. Dane AV, Schneider BH. Program integrity in primary and early secondary prevention: are implementation effects out of control? Clin Psychol Rev 1998;18:23-45.

89. Bumbarger B, Perkins D. After randomised trials: issues related to dissemination of evidence-based interventions. J Child Serv 2008;3:55-64. 
90. Stirman SW, Miller CJ, Toder K, et al. Development of a framework and coding system for modifications and adaptations of evidence-based interventions. Implement Sci 2013;8:65.

91. Gearing RE, El-Bassel N, Ghesquiere A, et al. Major ingredients of fidelity: a review and scientific guide to improving quality of intervention research implementation. Clin Psychol Rev 2011;31:79-88.

92. Raebel MA, Carroll NM, Kelleher JA, et al. Randomized trial to improve prescribing safety during pregnancy. J Am Med Inform Assoc 2007;4:440-50.

93. Goldzweig CL, Orshansky G, Paige NM, et al. Electronic health record-based interventions for improving appropriate diagnostic imaging: a systematic review and meta-analysis. Ann Int Med 2015;162:557-65.
94. Souza NM, Sebaldt RJ, Mackay JA, et al., for the CCDSS

Systematic Review Team. Computerized clinical decision support systems for primary preventive care: a decision-maker researcher partnership systematic review of effects on process of care and patient outcomes. Implement Sci 2011;6:87.

95. Hemens BJ, Holbrook A, Tonkin M, et al., for the CCDSS Systematic Review Team. Computerized clinical decision support systems for drug prescribing and management: a decision-maker-researcher partnership systematic review. Implement Sci 2011;6:89.

96. Docherty M, Smith R. The case for structuring the discussion of scientific papers. BMJ 1999;318:1224-5. 